\title{
The "Bag Breakup" Spume Droplet Generation Mechanism at High Winds. Part II: Contribution to Momentum and Enthalpy Transfer
}

\author{
YU. TROITSKAYA \\ Institute of Applied Physics, Nizhny Novgorod, and A. M. Obukhov Institute of Atmospheric Physics, Moscow, Russia \\ O. DRUZHININ AND D. KOZLOV \\ Institute of Applied Physics, Nizhny Novgorod, Russia
}

\section{S. ZILITINKEVICH}

Finnish Meteorological Institute, and Institute of Atmospheric and Earth System Research, University of Helsinki, Helsinki, Finland

(Manuscript received 30 May 2017, in final form 11 July 2018)

\begin{abstract}
In Part I of this study, we used high-speed video to identify "bag breakup" fragmentation as the dominant mechanism by which spume droplets are generated at gale-force and hurricane wind speeds. We also constructed a spray generation function (SGF) for the bag-breakup mechanism. The distinctive feature of this new SGF is the presence of giant $(\sim 1000 \mu \mathrm{m})$ droplets, which may significantly intensify the exchange between the atmosphere and the ocean. In this paper, Part II, we estimate the contribution of the bag-breakup mechanism to the momentum and enthalpy fluxes, which are known to strongly affect the development and maintenance of hurricanes. We consider three contributions to the spray-mediated aerodynamic drag: 1) "bags" as obstacles before fragmentation, 2) acceleration of droplets by the wind in the course of their production, and 3) stable stratification of the marine atmospheric boundary layer due to levitating droplets. Taking into account all of these contributions indicates a peaking dependence of the aerodynamic drag coefficient on the wind speed, which confirms the results of field and laboratory measurements. The contribution of the spray-mediated flux to the ocean-to-atmosphere moist enthalpy is also estimated using the concept of "reentrant spray," and the equation for the enthalpy flux from a single droplet to the atmosphere is derived from microphysical equations. Our estimates show that a noticeable increase in the enthalpy exchange coefficient at winds exceeding $30-35 \mathrm{~m} \mathrm{~s}^{-1}$ is due to the enhancement of the exchange processes caused by the presence of giant droplets originating from bag-breakup fragmentation.
\end{abstract}

\section{Introduction}

The effects of sea spray on heat, moisture, and momentum exchange between the atmosphere and the ocean have been the subject of numerous studies since pioneering works by Bortkovskii (1973), Borisenkov (1974), Wu (1974), Ling and Kao (1976), and Andreas et al. (1995). Modern studies are particularly motivated by the need to resolve problems formulated in a theoretical paper by Emanuel (1995), which showed the crucial role of the ratio of the enthalpy transfer coefficient to the surface drag coefficient, which should exceed 0.75 for realistic hurricane intensities with the

Corresponding author: Yuliya Troitskaya, yuliya@hydro.appl. sci-nnov.ru established parameters of the tropical atmosphere and ocean. The possibility of meeting this condition is indicated by meteorological (e.g., Powell et al. 2003; Holthuijsen et al. 2012; Takagaki et al. 2012, 2016) and oceanographic (Jarosz et al. 2007) observations, which demonstrate a nonmonotonous dependence of the drag coefficient on the wind speed, peaking at wind speeds of approximately $30-35 \mathrm{~m} \mathrm{~s}^{-1}$. There are also indications of an increase in the enthalpy exchange coefficient at hurricane winds (e.g., Richter et al. 2016; Bell et al. 2012). The influence of sea spray can be regarded as a plausible reason for both the reduction in the surface drag coefficient and the increase in the enthalpy transfer coefficient at hurricane winds. The corresponding reduction in turbulent stress is explained by Andreas (2004) and Kudryavtsev and Makin (2011) as the result 
of momentum exchange between droplets and airflow. Conversely, Makin (2005) and Kudryavtsev (2006) focused on the effect of the stable stratification of the marine atmospheric boundary layer by suspended spray droplets, in a similar way to work by Barenblatt and Golitsyn (1974). The efficiency of both mechanisms strongly increases with the size of the droplets and is proved to be most efficient for spume droplets (Kudryavtsev 2006; Andreas 2004; Kudryavtsev and Makin 2011). In accordance with the concept of reentrant spray proposed by Andreas and Emanuel (2001), these droplets also provide a large proportion of the spray-mediated enthalpy flux from the ocean to the atmosphere. The rate of production of sea spray is quantified by the spray generation function (SGF) $d F / d r$, which provides the number of spray droplets of a given radius produced per unit area of water surface per unit time. Empirically obtained SGFs can differ by six orders of magnitude in different observations [e.g., a compilation of experimental data by Andreas (2002)]. This difference results in significant uncertainties in estimates of the spray contribution to air-sea fluxes (e.g., Mueller and Veron 2014a,b).

In Troitskaya et al. (2018, hereafter Part I), we used high-speed video capture to show that, at wind speeds exceeding $20 \mathrm{~m} \mathrm{~s}^{-1}$, the dominant mechanism of spume droplet generation is "bag breakup" fragmentation. This process is similar to the fragmentation of liquid droplets and jets in gaseous flows (e.g., Gelfand 1996). Evidence of the presence of this spray-generating mechanism in a laboratory flume was first reported by Veron et al. (2012). The fragmentation starts with a small-scale elevation of the water surface, which then develops into a "microsail," that inflates into a water film bordered by a thicker rim (thus forming the "bag") that finally ruptures, producing hundreds of droplets. Recently (Troitskaya et al. 2017), we found that this phenomenon represents the dominant mechanism of spume droplet generation at high winds. Employing the general principals of statistical physics, in Part I we presented a statistical description of bag-breakup phenomena and used these statistics to derive the bagbreakup SGF, defined as the number of droplets of radius $r$ produced per unit area of the water surface per unit time. High-speed video shows that bags generate spray in two ways: (i) by rupturing the film of the inflated bag, which produces film droplets with average radii of about $100 \mu \mathrm{m}$, and (ii) by fragmenting the rim of the bag, thus yielding rim droplets with average radii in the range of $500-1000 \mu \mathrm{m}$ depending on the wind speed. Consequently, the SGF retrieved from our experiments was shown to be characterized by two peaks corresponding to the film and rim droplets, respectively. The rim-droplet peak located in the range $500-1000 \mu \mathrm{m}$ is a distinctive feature of the bagbreakup spray generation mechanism.

The amount of spray depends not only on wind but also on fetch, which drastically differs between laboratory and field conditions. We expressed the empirical dependence of SGFs on fetch via a dimensionless parameter called the windsea Reynolds number, $R_{\mathrm{WS}}=u_{*}^{2} / \nu \omega_{p}$, introduced by Toba and Koga (1986); here $\omega_{p}$ is the fetch-dependent peak frequency in the surface-wave spectra, $\nu$ is the air kinematic viscosity, and $u_{*}$ is the wind friction velocity. Based on the data from the laboratory experiments, in Part I we suggested two variants of empirical models for the fetch dependence of the specific number of bags and the related SGF. At high winds above $30 \mathrm{~m} \mathrm{~s}^{-1}$, both models predict similar values, while significant differences occur at winds below $30 \mathrm{~m} \mathrm{~s}^{-1}$ at fetches typical for open-ocean conditions.

In this paper, we estimate the contribution of the bagbreakup mechanism to both the enthalpy and momentum fluxes in the atmospheric boundary layer over the ocean, which are known to be of importance for the development and maintenance of hurricanes (cf. Emanuel 1995). In particular, we consider the contribution of giant droplets, which significantly modify spray-mediated airsea fluxes at hurricane winds compared to existing estimates based on conventional SGFs (e.g., Zhao et al. 2006; Andreas 1998; Fairall et al. 1994).

First, we estimate the impact of spray and bag breakup on the aerodynamic resistance of the water surface. We take three contributions into account. The first is the "droplet stress" caused by the acceleration of droplets by the wind in the course of their production from a small elevation in the water surface. This stress is almost completely provided by droplets originating from the fragmentation of the rim because of the dominant contribution of these rim droplets to the total droplet mass flux. The second contribution is the "bag stress" provided by bags (objects that look like microsails with typical sizes of $\sim 1 \mathrm{~cm}$ ), which act as obstacles to the near-water airflow. We also take into account the contribution related to the stable stratification in the near-surface airflow created by suspended droplets, which may also affect turbulence in the marine atmospheric boundary layer.

When estimating the effect of bag-breakup droplets on the moist enthalpy transfer from the ocean to atmosphere, we applied the concept of reentrant spray introduced by Andreas and Emanuel (2001), which considers the conservation of enthalpy in an air-water column. Here, we derive the spray-mediated enthalpy flux from equations of droplet microphysics developed by Pruppacher and Klett (1978), which also confirms the integral approach of Andreas and Emanuel (2001). Reentrant spray droplets that have cooled below the 
ambient air temperature due to evaporation of a small fraction of their volume fall back into the water and are the main contributors to the net ocean-atmosphere enthalpy flux. We show that these droplets are actually the giant "rim" droplets originating from bag-breakup fragmentation. We provide estimates of the enthalpy and sensible and latent heat fluxes using the approximate formulas derived by Andreas (2005). Currently, it is problematic to develop a more complex model, for example, similar to the stochastic Lagrangian models developed by Edson and Fairall (1994), Mueller and Veron (2014a), and Troitskaya et al. (2016). The most significant uncertainty is the distribution of the initial velocities of droplets ejected from the water surface. This strongly affects the motion of spray, especially the largest droplets, and the spray-mediated fluxes [cf. discussion of the momentum flux in Druzhinin et al. (2017) and Troitskaya et al. (2016)].

Our estimates provide only upper limits on the spraymediated enthalpy flux and exchange coefficient as they neglect the impact of the spray on the near-surface temperature and moisture profiles, thus reducing the feedback effect (see discussion by Bao et al. 2000, 2011; Bianco et al. 2011; Mueller and Veron 2014b).

The structure of the paper is as follows. Section 2 briefly describes the properties and statistics of droplets originating from the bag-breakup mechanism (discussed in detail in Part I) that are necessary for evaluating the spray-mediated fluxes. We estimate the contribution of the bag-breakup events to the aerodynamic resistance of the water surface in section 3 and discuss the effect of the stable stratification of the marine atmospheric boundary layer by suspended droplets in section 4 . In section 5 , we derive the spray-mediated fluxes using the heat balance equations for a single droplet formulated by Pruppacher and Klett (1978). Estimates of the contribution of the droplets to the air-sea enthalpy flux are provided in section 6. In all sections, the application of two models for the GFS fetch dependency is discussed. Section 7 presents a summary of the results.

\section{The bag-breakup SGF}

In this section, for convenience, we briefly summarize some properties of the statistics of bags that were revealed in Troitskaya et al. (2017) through postprocessing of high-speed video of the dynamics of the water surface under the action of high-speed airflow in a wind-wave flume.

We found that the specific number of bags $\langle N\rangle$ arising per unit time per unit area of the water surface (henceforth termed the specific number of bags) at different wind speeds can be related to the air friction velocity $u_{*}$ as

$$
\langle N\rangle=N_{0} u_{*}^{2} \exp \left(-\frac{U_{0}^{2}}{u_{*}^{2}}\right)
$$

The constants in Eq. (1) are as follows: $U_{0}=2 \mathrm{~m} \mathrm{~s}^{-1}$ with the $95 \%$ confidence interval between 1.87 and $2.13 \mathrm{~m} \mathrm{~s}^{-1}$, $N_{0}=9.27 \times 10^{2} \mathrm{~m}^{-4} \mathrm{~s}$ with the $95 \%$ confidence interval between $5.91 \times 10^{2}$ and $1.45 \times 10^{3} \mathrm{~m}^{-4} \mathrm{~s}$.

In Part I of this study, an alternative parameterization of the specific number of bags was derived based on the windsea Reynolds number introduced by Toba and Koga (1986):

$$
\operatorname{Re}_{B}=u_{*}^{2} / \omega_{p} \nu
$$

where $\omega_{p}$ is the peak frequency in the spectrum of surface wind waves and $\nu$ is the air kinematic viscosity. Parameter $\operatorname{Re}_{B}$ can be expressed via the wave-age parameter $\Omega$, defined as the ratio of the phase velocity $c_{p}$ of the surface waves at the peak frequency in the wind-wave spectrum and wind speed at a reference height of $10 \mathrm{~m} U_{10}, \Omega=c_{p} / U_{10}$ :

$$
\mathrm{Re}_{B}=U_{10}^{3} C_{D} / g \Omega \nu .
$$

Here $C_{D}=u_{*}^{2} / U_{10}^{2}$ is the aerodynamic drag coefficient of the water surface and $g$ is gravitational acceleration. The specific number of bags can be expressed in terms of $\operatorname{Re}_{B}$ as

$$
\langle N\rangle=M_{0} \operatorname{Re}_{B}^{3 / 2} \exp \left(-\frac{M_{1}}{\operatorname{Re}_{B}^{3 / 2}}\right) .
$$

The best fit to the experimental data in Part I gave the following constants for Eq. (2): $M_{0}=2.58 \times 10^{-4} \mathrm{~m}^{-2} \mathrm{~s}^{-1}$ with the $95 \%$ confidence interval between $2.22 \times 10^{-4}$ and $3.00 \times 10^{-4} \mathrm{~m}^{-2} \mathrm{~s}^{-1}$, and $M_{1}=6.93 \times 10^{5}$ with the $95 \%$ confidence interval between $6.22 \times 10^{5}$ and $7.64 \times$ $10^{5}$. The coefficient of variation of the specific number of bags defined by Eqs. (1) or (2) is approximately $25 \%$.

We also determined bag characteristics such as radius at the moments of nucleation and film rapture, $R_{1}$ and $R_{2}$, respectively, and the time between these two moments (i.e., the bag lifetime) $\tau$ and also the correlation $\langle\bar{R} \tau\rangle$ between $\tau$ and the mean radius of the bag, $\bar{R}=$ $\left(R_{1}+R_{2}\right) / 2$ (cf. Fig. 1a):

$$
\begin{aligned}
\left\langle R_{1}\right\rangle\left(u_{*}\right) & =5.9 u_{*}^{-1}, \\
\left\langle R_{2}\right\rangle\left(u_{*}\right) & =9.6 u_{*}^{-1}, \\
\langle\tau\rangle\left(u_{*}\right) & =7.7 u_{*}^{-2}, \quad \text { and } \\
\langle\bar{R} \tau\rangle & =64.4 u_{*}^{-3},
\end{aligned}
$$

where $u_{*}$ is expressed in metric units, $\left\langle R_{1}\right\rangle$ and $\left\langle R_{2}\right\rangle$ in millimeters, and $\langle\tau\rangle$ in milliseconds. The standard 


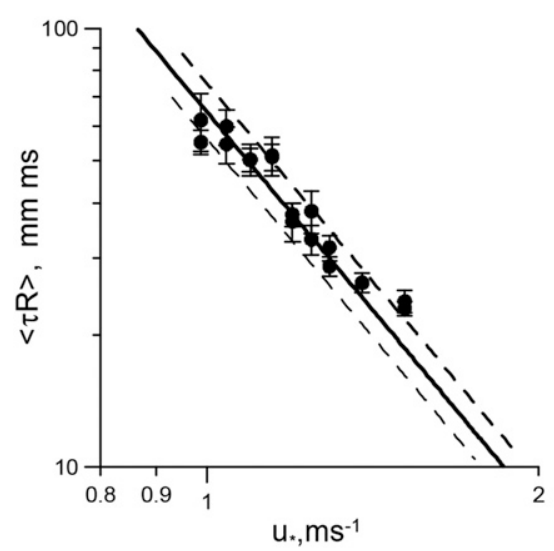

(a)

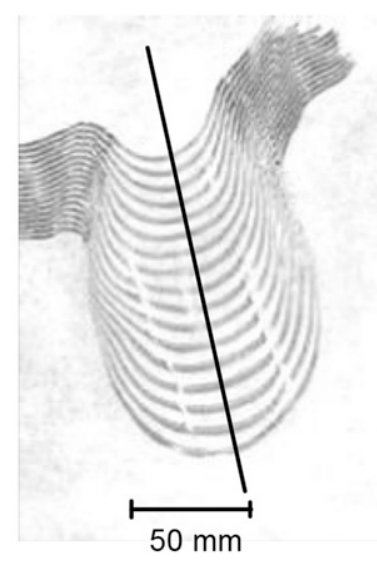

(b)

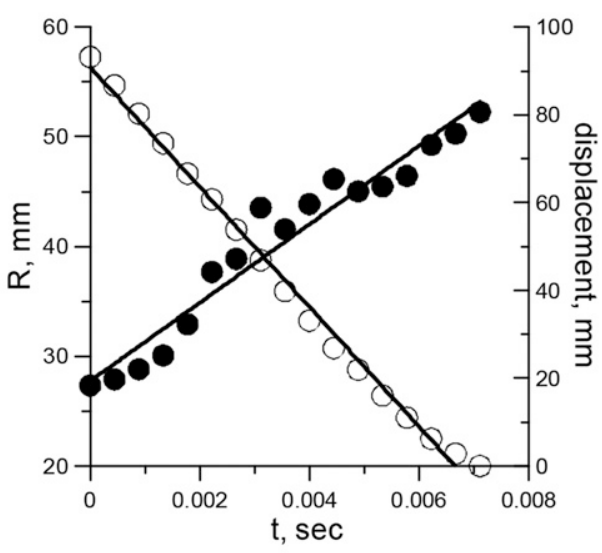

(c)

FIG. 1. (a) Parameter $\langle\tau R\rangle$ vs wind friction velocity $u_{*}$. The error bars depict the mean-square error of the expected value, the solid curve is approximated by Eq. (6), and the dashed curves show the uncertainty of the approximation. (b) Displacement of the inflating canopy of the bag (top view) obtained by superimposition of a sequence of frames of high-speed video taken with an interval of $4.5 \mathrm{~ms}$. (c) Displacement of the canopy of the bag from top to bottom along the line in panel (b) (open circles) and the canopy radius (closed circles) vs time.

deviation of the coefficients in Eqs. (3)-(6) is approximately $13 \%$. Notice that comparing Eqs. (4)-(6) suggests $\langle\bar{R} \tau\rangle \approx 0.87\langle\bar{R}\rangle\langle\tau\rangle$.

As discussed in section 1, droplet production occurs in two ways during bag breakup: (i) by rupturing the film of the inflated bag, producing film droplets, and (ii) by fragmentation of the rim, producing rim droplets. We estimated the magnitude of these two contributions by using available data on the size distributions of droplets formed during the rupture of a submerged bubble (Lhuissier and Villermaux 2012), fragmentation of liquid droplets in gaseous flows (Chou and Faeth 1998), and fragmentation of liquid ligaments (Marmottant and Villermaux 2004). The resulting SGF can be expressed as

$$
\begin{aligned}
\frac{d F\left(r, u_{*}\right)}{d r}= & \langle N\rangle\left[\frac{A_{1}}{L}\left(\frac{\rho_{w} g L^{2}}{\sigma}\right)^{1.18}\left(\frac{r}{\theta}\right)^{7.3} e^{-5.2 \sinh \left(\frac{3}{7} \ln \frac{r}{\theta}\right)}\right. \\
& \left.+A_{2} \frac{N_{\text {rim_drops }}}{\Theta}\left(\frac{r}{\Theta}\right)^{4.5} e^{-3.94 \sinh \left(\frac{1}{2} \ln \frac{r}{\Theta}\right)}\right] .
\end{aligned}
$$

Here, $A_{1}=3.3 \times 10^{-9}, A_{2}=1.5 \times 10^{-4}$ are dimensionless constants, $L=20 \mathrm{~m}$, and $N_{\text {rim_drops }}=8.3$. The scales $\theta=0.001\left[\left\langle R_{2}\right\rangle\left(u_{*}\right)\right]^{4 / 3} / L^{1 / 3}$ and $\Theta=0.0021\left\langle R_{2}\right\rangle\left(u_{*}\right)$ are prescribed by the average radius of the bag at the moment of its rupture $\left\langle R_{2}\right\rangle\left(u_{*}\right)$, defined by Eq. (4). Note that $\left\langle R_{2}\right\rangle\left(u_{*}\right)$ decreases with increasing wind speed, and it also follows from the SGF, Eq. (7), that the average droplet size prescribed by bag size decreases with the wind speed. Parameter $\langle N\rangle$ in Eq. (7) is defined by Eqs. (1) or (2). The coefficient of variation of the SGF, Eq. (7), as well as $\langle N\rangle$, is approximately $25 \%$.

To estimate the effect of the bag-breakup spray production mechanism in the ocean, we have to take into account the dependence of the amount of spray not only on wind but also on fetch, which differs drastically between laboratory and field conditions. Using Eq. (2) for the specific number of bags provides the universal SFG, which can be used directly both in the laboratory and the field if the appropriate wave-age parameter $\Omega$ for field conditions is used.

Another method used in Part I to adjust our laboratory data to field conditions is the simple rescaling of the specific number of bags by the $\mathrm{Re}_{B}^{3 / 2}$ dependence of the spray production rate, based on the results of Zhao et al. (2006). Taking the fixed number of droplets that are produced by one bag into account, and making the assumption that the number of bags under field and laboratory conditions has a similar relation to that of the field and laboratory spray generation functions, in Part I we obtained

$$
\langle N\rangle=N_{0} \frac{u_{*}^{2}}{U_{0}^{2}}\left(\frac{12.4 u_{*}^{0.5}}{g \Omega \sqrt{C_{D 10}}}\right)^{1.5} \exp \left(-\frac{U_{0}^{2}}{u_{*}^{2}}\right) .
$$

All variables in Eq. (8) are expressed in metric units. The characteristics of the dependencies of the specific number of bag-breakup events given by Eq. (2) and Eq. (8) are discussed in Part I. Equation (2) is shown to predict more events at lower wind speeds at fetches 
typical for open-ocean conditions and slightly faster growth in the number of events with wind speed in comparison with Eq. (8).

The wave-age parameter is a good means of defining fetch in the rotating and displacing wind fields typical of hurricanes. Direct measurements under hurricane conditions by Wright et al. (2001) yielded $\Omega=2.5-3.5$. We use this parameter below to estimate fluxes and exchange coefficients under field conditions.

Note that Eqs. (2) and (8) were obtained on the basis of a limited dataset obtained in a laboratory experiment in a straight channel with a very short wind fetch. Currently, sufficient data are not available to allow confident extrapolation to field conditions, and so we cannot favor one of these expressions over the other. Below, we compare the estimates of fluxes and exchange coefficients obtained by using each of them.

\section{Contribution of bag-breakup events to the aerodynamic resistance of the water surface at hurricane winds}

Let us now estimate the contribution of bag-breakup events to the momentum flux in the marine atmospheric boundary layer. This contribution is determined by two components, both working to increase the surface drag coefficient. The first is the surface stress, or the bag stress $F_{M b}$, provided by the canopies of bags with typical sizes of $\sim 1 \mathrm{~cm}$ forming obstacles to the near-water wind flow. The second component is the spray-mediated momentum flux, or the droplet stress $F_{M d}$, determined by the momentum acquired by droplets in the course of their production. Both components are estimated below.

\section{a. The bag stress}

To estimate the contribution of the canopies of the bags to the aerodynamic resistance of the water surface, we need to determine the drag force imposed by each canopy on the surrounding airflow. The analysis of individual frames of high-speed video allowed us to investigate the temporal evolution of bags as well as their characteristic geometric dimensions. In particular, it was revealed that both the canopy radius and the translation of the entire bag in the process of its evolution are linear functions of time (Figs. 1b,c). Since the velocity with which the bag advances under the drag of the airflow is constant, there is equilibrium between the air pressure difference on opposite sides of the canopy of the bag and the surface tension of the inflated film.

Let us use a simplified model, treating the canopy of the bag as a water-film hemisphere with radius $R(t)$ maintained by the balance between the aerodynamic pressure force and the surface tension. The latter is determined by the difference in capillary forces acting on the outer convex and inner concave surfaces. Thus, the balance condition can be expressed as

$$
\left.\left(p_{1}-p_{2}\right)\right|_{S}=\frac{4 \sigma}{R(t)}
$$

where $p_{1}$ and $p_{2}$ are the dynamic pressure at the inner and outer sides of the bag's canopy and $\sigma$ is the surface tension coefficient.

The instantaneous force imposed on the bag by the airflow, and vice versa, can then be evaluated as

$$
\mathbf{f}(t)=\iint_{S}\left(p_{1}-p_{2}\right) \mathbf{r}_{0} d s=\iint_{S} \frac{4 \sigma}{R(t)} \mathbf{r}_{0} d s=4 \pi \sigma R(t) \mathbf{x}_{0} .
$$

Here, $\mathbf{r}_{0}$ is the unit radial vector and $\mathbf{x}_{0}$ is the unit vector in the direction of canopy inflation close to the wind direction.

Now suppose that at time $t$ there are $\Delta N(t)$ bags at the water surface area $\Delta S$ and each $i$ th bag acts on the airflow with force $\mathbf{f}_{i}(t)$. The total force acting on the airflow from this area can then be evaluated as a vector sum of the forces caused by individual bags. The projection of this force to the wind direction is $\Delta F(t)=\sum_{i=1}^{\Delta N(t)} f_{i}(t)$, where $f_{i}(t)$ is the along-wind component of $\mathbf{f}_{i}(t)$. Since the direction of $\mathbf{f}_{i}(t)$ is close to the wind direction, $f_{i}(t) \approx\left|\mathbf{f}_{i}(t)\right|$. As this total force fluctuates, we perform averaging over a time interval $\Delta T$ that is much larger than the bag lifetime [cf. Eq. (4)] and obtain a mean drag force:

$$
\begin{aligned}
\langle\Delta F(t)\rangle & =\frac{1}{\Delta T} \int_{t}^{t+\Delta T} \sum_{i=1}^{\Delta N(t)} f_{i}(t) d t \\
& =4 \pi \sigma \frac{1}{\Delta T} \int_{t}^{t+\Delta T} \sum_{i=1}^{\Delta N(t)} R_{i}(t) d t .
\end{aligned}
$$

The contribution of each $i$ th bag to the sum under the integral on the right-hand side of Eq. (9) can be evaluated as

$$
\int_{t}^{t+\Delta T} R_{i}(t) d t=\bar{R}_{i} \tau_{i},
$$

where $\bar{R}_{i}$ is the average radius of the bag canopy during its lifetime from the moment of nucleation to the moment of rupture $\tau_{i}$. Figure $1 \mathrm{c}$ shows that $\bar{R}_{i}$ is a linear function of time, so that $\bar{R}_{i}=\left(R_{1 i}+R_{2 i}\right) / 2$, where $R_{1 i}$ and $R_{2 i}$ are the $i$ th canopy radii in the moments of nucleation and rupture, respectively. Equation (9) can be used to determine the average drag force imposed by the bags on the airflow per unit area in the form: 


$$
F_{M b}=\frac{\langle\Delta F(t)\rangle}{\Delta S}=4 \pi \sigma\langle\bar{R} \tau\rangle\langle N\rangle,
$$

where $\langle N\rangle$ is the average number of bags produced per unit area per unit time described by Eq. (2) or Eq. (8). More precisely, in this way we obtain an upper estimate for the force acting on the bag since some of the bag canopies are spherical segments. However, this does not violate the scaling used and can only affect the coefficient value.

Substituting Eq. (6) for $\langle\bar{R} \tau\rangle$ and Eq. (8) or Eq. (2) in Eq. (10) yields, respectively,

$$
F_{M b}=4 \pi \sigma N_{0} \frac{u_{*}^{2}}{U_{0}^{2}}\left(\frac{12.4 u_{*}^{0.4}}{g \Omega \sqrt{C_{D 10}}}\right)^{1.5} e^{-U_{0}^{2} / u_{*}^{2}} \frac{\lambda}{u_{*}^{3}},
$$

and

$$
F_{M b}=4 \pi \sigma M_{0} \operatorname{Re}_{B}^{3 / 2} \exp \left(-\frac{M_{1}}{\operatorname{Re}_{B}^{3 / 2}}\right) e^{-U_{0}^{2} / u_{*}^{2}} \frac{\lambda}{u_{*}^{3}} .
$$

Comparison of Figs. $2 \mathrm{~b}$ and $2 \mathrm{~d}$ shows that the dependencies of $F_{M b}$ on wind velocity calculated using Eq. (11) and Eq. (12) are similar at $U_{10}>30 \mathrm{~m} \mathrm{~s}^{-1}$, but at lower winds Eq. (12) predicts a larger contribution of the bags to the surface stress than Eq. (11). The tangential stress $F_{M b}$ is determined by the specific number of bags, on the one hand, and their dimensions and lifetimes, on the other. With increasing wind speed, these factors have opposite tendencies: an increase in the number of bags and a decrease in their size and lifetime. As a result, $F_{M b}$ has a tendency to saturation with increasing wind speed. Using Eq. (11) predicts faster saturation of the surface stress with wind speed than Eq. (12). The differences in the dependency of $F_{M b}$ on wind speed in Eq. (12) and Eq. (11) result from the peculiarities of the approximations in Eq. (2) and Eq. (8), namely, that Eq. (2) predicts a higher number of bag-breakup events at lower winds at fetches typical for open-ocean conditions and faster growth in the number of events with wind speed.

\section{b. The droplet stress}

The contribution of spray to the surface stress, the droplet stress $F_{M d}$, is determined by the momentum that droplets acquire when being accelerated by the airflow during their development from a small perturbation at the water surface (before the droplets has been formed) through the stage of a bag to droplets. The contribution of droplets of a certain radius $r$ to the surface stress is

$$
P=\frac{4 \pi r^{3}}{3} \rho_{w} u \frac{d F\left(r, u_{*}\right)}{d r},
$$

where $u$ is the final velocity of the droplet and $\rho_{w}$ is the droplet density. To be more precise, in the beginning of the process, the velocity of the volume of water, which later is transformed to droplets is equal to the wave orbital velocity, assumed here to be negligibly small. Equation (13) shows that $P$ is proportional to the SGF as the mass flux, which has a sharp peak at sizes relevant for the giant droplets originating from fragmentation of the rim during bag breakup (see Figs. 2a,c). The velocities of the giant rim droplets can be estimated as the velocities of the bags' edges $\langle u\rangle$. This value was directly measured by postprocessing of a sequence of video frames [the details of the algorithm can be found in the supplement to Troitskaya et al. (2017) and in Part I of this study], and the best fit of $\langle u\rangle$ on the air friction velocity is

$$
\langle u\rangle=u_{0}+\Gamma u_{*},
$$

where $u_{0}=1.96 \pm 0.44 \mathrm{~m} \mathrm{~s}^{-1}$ and $\Gamma=1.21 \pm 0.36$.

Given that the rim droplet velocities are independent of their size and equal to the bag rim velocity, the spraymediated momentum flux can be expressed as

$$
F_{M d}=\rho_{w} \int_{0}^{\infty} \frac{d F\left(r, u_{*}\right)}{d r} \frac{4 \pi r^{3}}{3}\langle u\rangle d r
$$

where $\langle u\rangle$ is defined by Eq. (14).

Strictly speaking, Eq. (15) gives an upper bound estimate of the contribution of droplets to the surface drag because we neglect the portion of the momentum flux that a droplet can give back to the atmosphere during gravitation settling as its velocity is adjusted to the decreasing local airflow velocity. However, we do not expect this to have a strong effect because of the high inertia of the giant droplets that contribute most to the momentum flux. With an increase in the size of the droplet, there will be a simultaneous increase in the time for droplet velocity adjustment to the velocity of the surrounding airflow and decrease in the time of residence of the droplet in the atmosphere due to the increase of the gravitational settling velocity. The quantitative estimates in the appendix show that the residence time is less than the adjustment time for droplets with radii above $150 \mu \mathrm{m}$ for a wind speed of $20 \mathrm{~m} \mathrm{~s}^{-1}$, above $300 \mu \mathrm{m}$ for a wind speed of $40 \mathrm{~m} \mathrm{~s}^{-1}$, and above $450 \mu \mathrm{m}$ for a wind speed of $60 \mathrm{~m} \mathrm{~s}^{-1}$ (see Fig. A1a). This means that droplets with larger radii do not have time to transfer the momentum accumulated in the entire process of production back to the wind flow during the residence time and this effect can be neglected. Comparison with Fig. 2a allows us to conclude that our assumption is correct up to a wind speed of $40 \mathrm{~m} \mathrm{~s}^{-1}$, when the peak contribution to the momentum 


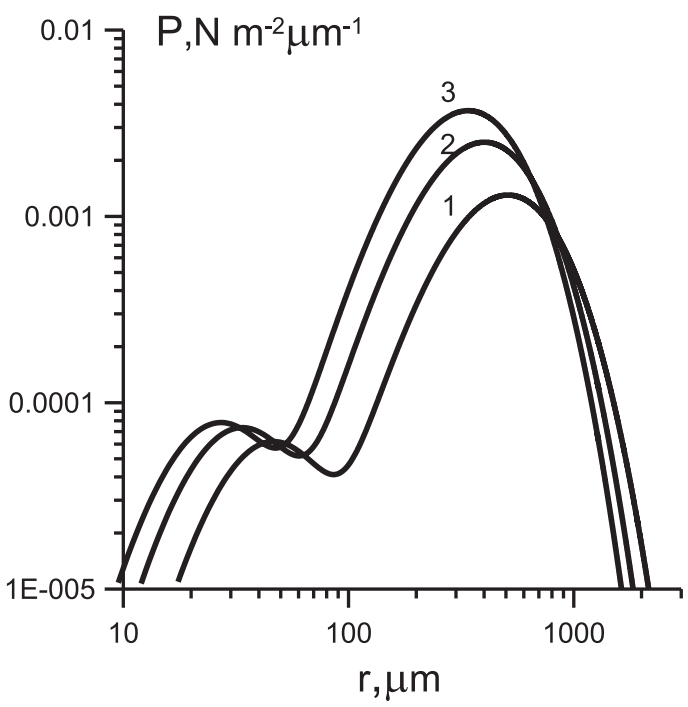

(a)

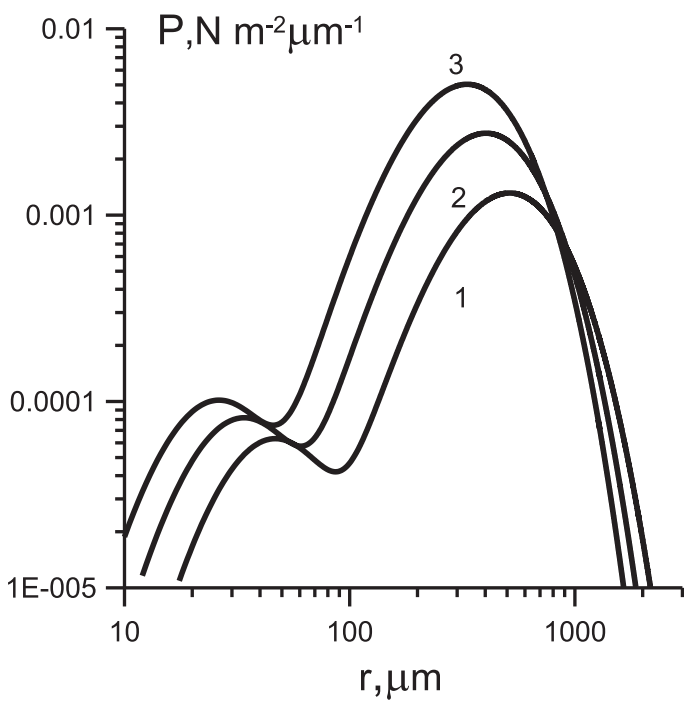

(c)

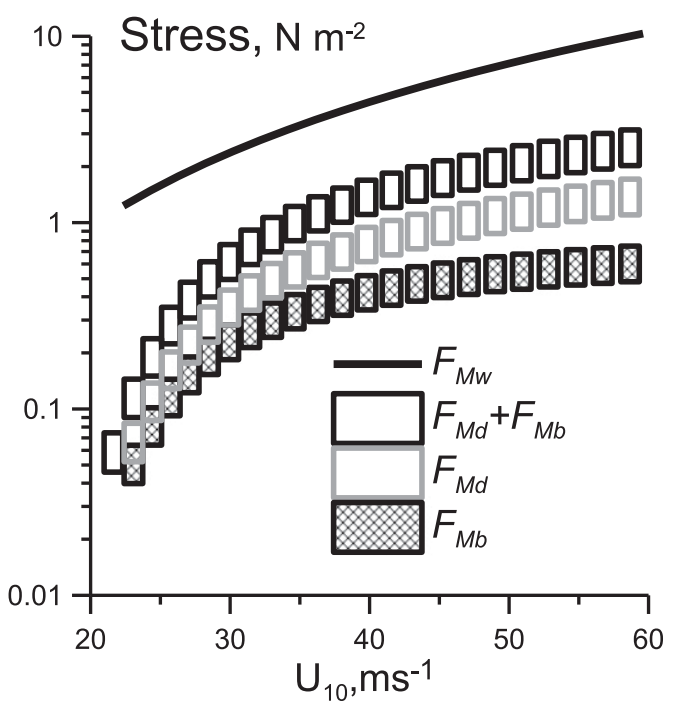

(b)

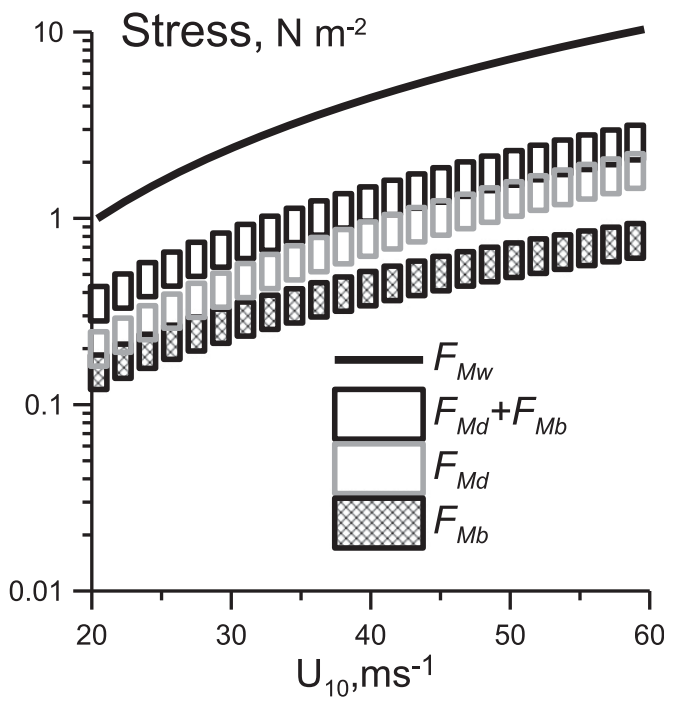

(d)

FIG. 2. Contribution of bag breakup to air-sea momentum exchange: plots of calculations using (top) Eq. (8) and (bottom) Eq. (2) for the specific number of bags. (a),(c) Partial contributions of droplets produced by the bagbreakup mechanism to the momentum flux $P$. Values of the $10-\mathrm{m}$ wind speed $U_{10}$ are $40 \mathrm{~m} \mathrm{~s}^{-1}$ (marker 1 ), $50 \mathrm{~m} \mathrm{~s}^{-1}$ (marker 2), and $60 \mathrm{~m} \mathrm{~s}^{-1}$ (marker 3); the wave-age parameter $\Omega=2.5$. (b),(d) Contributions to the surface stress caused by bags $F_{M b}$ [Eq. (14)], droplets $F_{M d}$ [Eq. (16)], and direct turbulent transfer $F_{M w}$ [Eq. (17)] vs 10-m wind speed $U_{10}$. The vertical bars depict variations in the wave-age parameter $\Omega$ from 2.5 to 3.5.

flux corresponds to droplets with a radius of approximately $500 \mu \mathrm{m}$, but that it may be broken at higher wind speeds.

As for $F_{M b}$, the dependence of $F_{M d}$ on the wind speed is determined by two opposite tendencies: on the one hand, the increase in the number of droplets [cf. Eq. (7)], and on the other, the decrease in droplet size proportional to the average bag size, which decreases with increasing wind speed according to Eq. (4). As with $F_{M b}$, using Eq. (2) for the specific number of bags in Eq. (7) for the SGF gives a faster increase in the number of droplets with wind speed than using Eq. (8), and accordingly, the latter indicates more rapid saturation of the surface stress with increasing wind speed. Besides, as with the bag stress, lower droplet stress at winds below $30 \mathrm{~m} \mathrm{~s}^{-1}$ is predicted with Eq. (8). The peculiarities 


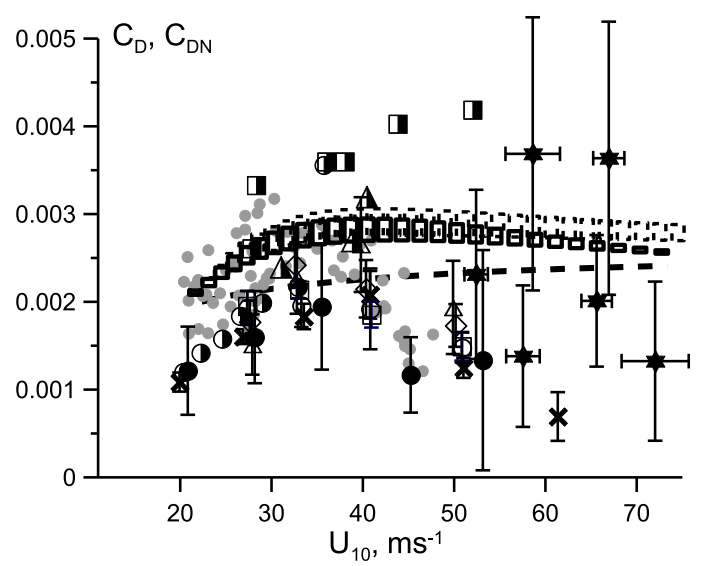

(a)

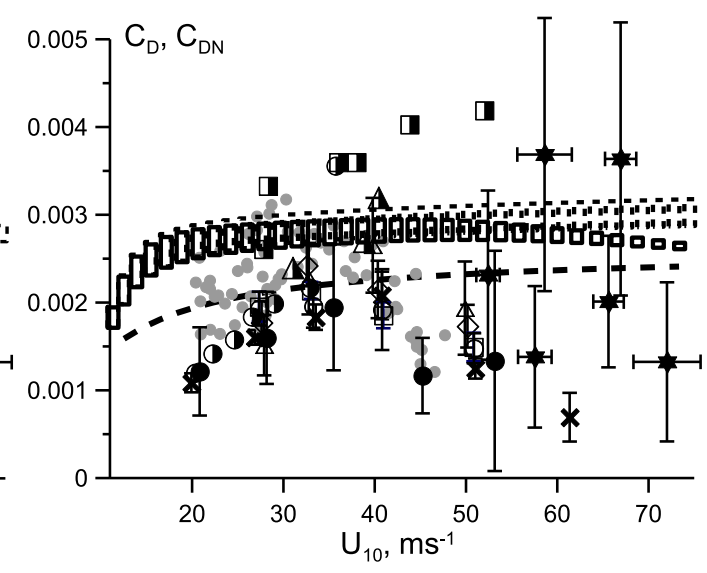

(b)

FIG. 3. Surface drag coefficient $C_{D}$ vs $U_{10}$ under our stress model. Dashed curve shows $C_{D}$ from Foreman and Emeis (2010). Experimental data: squares, diamonds, triangles, and circles are from Powell et al. (2003), crosses are from Holthuijsen et al. (2012), asterisks are from Bell et al. (2012), closed circles are from Richter et al. (2016), gray dots are from Jarosz et al. (2007), half-closed circles are from Hawkins and Rubsam (1968), half-closed triangles are from Miller (1962), and half-closed squares are from Miller (1964). The dashed line floating bars mark $C_{D N}\left(U_{10 N}\right)$ in a neutrally stable atmosphere, and the solid line floating bars mark $C_{D}\left(U_{10}\right)$ when the effect of the buoyancy of the droplets is taken into account. The vertical bars depict variations in the wave-age parameter $\Omega$ from 2.5 to 3.5. Calculations use (a) Eq. (8) and (b) Eq. (2) for the specific number of bags.

described can be seen by comparing the corresponding curves in Figs. 2b and 2d.

We assume that the total turbulent shear stress at hurricane winds is determined by three dominant factors: the form drag of the waves $F_{M w}$, the spraymediated momentum flux $F_{M d}$ in Eq. (15), and the aerodynamic resistance of bags $F_{M b}$ in Eqs. (11) and (12):

$$
F_{M}=F_{M w}+F_{M d}+F_{M b} .
$$

The contribution of form drag by surface waves $F_{M w}$ at storm and hurricane winds is rather uncertain (e.g., Soloviev et al. 2014, and references therein). Here it is approximated by the empirical drag law derived by Foreman and Emeis (2010) from data acquired at winds below $25 \mathrm{~m} \mathrm{~s}^{-1}$, when the effect of the bags is obviously negligible, and surface resistance is determined by the form drag from long gravity waves to ripples. The equation for $F_{M w}$ is as follows:

$$
F_{M w}=\rho_{a}\left[0.051\left(U_{10}-8\right)+0.27\right]^{2} .
$$

According to Eq. (16), the form drag tends to a constant number $\approx 0.0026$. The drag coefficient for a neutrally stable atmospheric boundary layer can be found by definition

$$
C_{D N}=F_{M} /\left(\rho_{a} U_{10}^{2}\right)
$$

Here, using the notation $C_{D N}$ emphasizes that it describes the neutral drag coefficient in contrast to $C_{D}$, used in the next section, which takes into account the effect of the stable stratification of the marine atmospheric boundary layer by suspended droplets. The bag-related resistance and the momentum acquired by droplets in the course of their formation both lead to an increase in the surface drag coefficient. Using the two models for the dependency of the specific number of bags on fetch, Eq. (2) and Eq. (8), yields qualitatively different functions for $C_{D N}\left(U_{10}\right)$. The tendency to relatively rapid saturation of the "bag and spray" momentum flux provided by Eq. (8) leads to a slightly peaking wind speed dependence of $C_{D N}$ (Fig. 3a), while using Eq. (2) leads to only saturating dependency for $C_{D N}\left(U_{10}\right)$ within the range of wind speeds being considered (Fig. 3b); however, the quantitative difference between the two models is only about $8 \%$.

\section{The effect of stable stratification of the marine atmospheric boundary layer by suspended droplets}

To evaluate the effect of suspended droplets by creating a stable stratification of the marine atmospheric boundary layer, we apply the approach suggested by Bao et al. (2011) and Kudryavtsev (2006) and also take the droplet size distribution into account. It should be 
mentioned that the injection of large droplets into the airflow is markedly intermittent in space and time since it occurs near the crests of steep and breaking waves. To estimate the effect of droplets on the mean flow in the turbulent boundary layer, we will use averaging over a time period far exceeding the period of the dominant surface waves. Similarly to Bao et al. (2011) and Kudryavtsev (2006), we suppose that the spray droplets are injected at height $z_{s}$; above this level, the size-dependent droplet concentration obeys the steady equation of conservation:

$$
K_{s}(z, r) \frac{\partial S(z, r)}{\partial z}+V_{f}(r) S(z, r)=0 .
$$

In Eq. (17), $S(z, r)$ is the time-averaged volume concentration of droplets of radius $r, K_{S}(z, r)$ is the sizedependent eddy diffusivity of droplet concentration, and $V_{f}(r)$ is the gravitational settling velocity of the droplets. For small droplets with $V_{f}(r)$ below the friction velocity in the boundary layer flow, which can be considered as passive tracers, $K_{S}$ is assumed equal to the eddy viscosity coefficient similarly to Bao et al. (2011). Here, the model is also applied to large droplets, for which the effect of turbulent diffusion is substantially reduced. The effect of the reduction of the turbulent diffusion is described by the dependence of eddy diffusivity on the droplet size [see Eq. (21) below]. Note that the model can be used only for rough estimates of the effect of suspended droplets. However, there is no need to develop a more sophisticated model because the effect of suspended droplets gives a deflection in the exchange coefficients of about $7 \%-8 \%$ (see below), which is minor compared to more substantial effects such as the effect on the surface drag coefficient of the aerodynamic resistance of the bags as obstacles or the inertial effects of the large droplets (discussed in sections $3 a$ and $3 b$ ).

Following Bao et al. (2011) and Kudryavtsev (2006), we assume that the level of spray injection, $z=z_{s}$, coincides with the level of wave breaking. Similarly to Iida et al. (1992), we take $z_{s}$ equal to the fraction of the significant wave height $H_{s}$, namely,

$$
z_{s}=0.635 H_{s} ; \quad H_{s}=0.018 \Omega^{-1.5} U_{10}^{2} / g .
$$

Below $z_{s}$, the time-averaged concentration of droplets is constant, and the balance between the up and down fluxes of droplets gives the boundary condition for Eq. (17) [see a more detailed discussion by Veron (2015)]:

$$
\left.S(z, r)\right|_{z=z_{s}}=\frac{4}{3} \pi r^{3} \frac{d F}{d r} \frac{1}{V_{f}(r)} .
$$

We use the Stokes terminal velocity of a spherical drop with the finite Reynolds number for $V_{f}(r)$, although in a turbulent flow it can differ [cf. Veron (2015) for details]. We applied software developed by Andreas (2013) to perform the calculations. Like Bao et al. (2011) and Kudryavtsev (2006), we use the following steady equation for the average momentum budget:

$$
\frac{d U}{d z}=\frac{u_{*}}{\kappa z} \Phi_{M}\left(\frac{z}{L}\right),
$$

where $L$ is an analog of the Monin-Obukhov (MO) scale introduced for describing the effect of suspended droplets on the static stability:

$$
L=\frac{u_{*}^{3}}{\kappa g\left(\rho_{w} / \rho_{a}\right)\langle s w\rangle},
$$

where $\kappa=0.4$ is the Von Kármán constant, $\rho_{w} / \rho_{a}$ is the ratio of water and air densities, and $\langle s w\rangle$ is the flux of the volume concentration of droplets integrated over their sizes:

$$
\langle s w\rangle=-\int K_{s}(z, r) \frac{\partial S(z, r)}{\partial z} d r=\int V_{f}(r) S(z, r) d r .
$$

Similarly to the original MO scale introduced for the stratified turbulent shear flow, the length $L$ is the height to which the production of turbulent kinetic energy by shear exceeds its suppression by the stable stratification provided by suspended droplets.

Like Bao et al. (2011) and Kudryavtsev (2006), we used the following approximation for $\Phi_{M}$ :

$$
\Phi_{M}\left(\frac{z}{L}\right)=1+b \frac{z}{L}
$$

where $b=5$ (see, e.g., Dyer 1974). Equation (19) can then be rewritten

$$
\frac{d U}{d z}=\frac{u_{*}}{\kappa z}\left[1+b \frac{\rho_{w} \kappa g z}{\rho_{a} u_{*}^{3}} \int V_{f}(r) S(z, r) d r\right] .
$$

From Eq. (20) we define the eddy viscosity coefficient as

$$
K_{m}(z)=\frac{u_{*} \kappa z}{\left[1+b \frac{\rho_{w} \kappa g z}{\rho_{a} u_{*}^{3}} \int V_{f}(r) S(z, r) d r\right]} .
$$

The expression for the eddy diffusivity coefficient for droplets was taken from Rouault et al. (1991):

$$
K_{s}(z, r)=\frac{K_{s 0}(z)}{1+C_{2}\left[V_{f}^{2}(r) / 1.56 u_{*}^{2}\right]},
$$


where $K_{s 0}(z)$ is the eddy diffusivity of a passive tracer and $C_{2}=5$ is an empirical constant defined by Rouault et al. (1991) on the basis of available experimental data in the marine atmospheric boundary layer. This model, Eq. (22), assumes a reduction of the turbulent diffusivity of massive particles due to their inertia. For $u_{*}=2 \mathrm{~m} \mathrm{~s}^{-1}$ and $r=1000 \mu \mathrm{m}$, Eq. (22) predicts an approximately 50 -fold reduction of the eddy viscosity coefficient in comparison with passive tracers.

Under the assumption that the eddy diffusivity of a passive tracer equals the eddy viscosity, $K_{s 0}(z)=K_{m}(z)$, Eq. (17) can be rewritten as

$$
\begin{gathered}
\frac{\partial S(z, r)}{\partial z}+\frac{V_{f}(r)}{\kappa u_{*} z} S(z, r)\left\{1+C_{2}\left[V_{f}^{2}(r) / 1.56 u_{*}^{2}\right]\right\} \\
\times\left[1+\beta \frac{\rho_{w} \kappa g z}{\rho_{a} u_{*}^{3}} \int V_{f}\left(r_{1}\right) S\left(z, r_{1}\right) d r_{1}\right]=0 .
\end{gathered}
$$

Integrating Eq. (23) from $z_{s}$ to a current $z$ and taking into account the uniform time-averaged spray concentration at $z<z_{s}$, (cf. Veron 2015) yields

$$
U(z)=\frac{u_{*}}{\kappa} \ln \frac{z}{z_{0}}+\beta \frac{\rho_{w} g}{\rho_{a} u_{*}^{2}} \int_{z_{s}}^{z} d z_{1} \int V_{f}(r) S\left(z_{1}, r\right) d r .
$$

Here, $z_{0}$ can be directly expressed via the drag coefficient for a neutrally stable atmospheric boundary layer to provide an alternative definition:

$$
z_{0}=H_{10} e^{-\kappa / \sqrt{C_{D N}}}
$$

where $H_{10}$ is the $10-\mathrm{m}$ height.

Finally, the drag coefficient $C_{D}$, modified by suspended droplets, becomes

$$
C_{D}=\frac{C_{D N}}{\left[1+\beta \frac{\rho_{w} g}{\rho_{a} u_{*}^{2}} \sqrt{C_{D N}} \int_{z_{s}}^{\infty} d z_{1} \int V_{f}(r) S\left(z_{1}, r\right) d r\right]^{2}} .
$$

Figures $3 \mathrm{a}$ and $3 \mathrm{~b}$ present the predictions for $C_{D}$ through Eq. (24) for the two models of the dependence of the SGF on the wind fetch. It can be seen that the effect of the stable stratification of the atmosphere due to the presence of droplets is not strong and results in only an approximately $7 \%-8 \%$ decrease in the surface drag coefficient. It also results in a decrease in $C_{D}$ at very high winds and a peaking dependence, $C_{D}\left(U_{10}\right)$, for both models. Thus, we can conclude that the comprehensive model, which includes the triple effect of the form drag of bags, the spray inertia, and the density stratification of the airflow by suspended droplets can provide the peaking function $C_{D}\left(U_{10}\right)$.

\section{Heat and enthalpy fluxes from droplets to the atmosphere}

According to Emanuel (1995), hurricane intensity is controlled by the net moist enthalpy transfer from the ocean to the atmosphere rather than by the separate contributions of the sensible and latent heat fluxes. The sea spray contribution to the net air-sea enthalpy flux is recognized by Andreas and Emanuel (2001) in the concept of reentrant spray. Assuming the conservation of the overall enthalpy in an air-water column, Andreas and Emanuel (2001) showed that droplets completely evaporated to the atmosphere do not contribute to the net enthalpy flux between the atmosphere and ocean. For these droplets, the latent heat of evaporation is exactly compensated for by the sensible heat extracted by the spray from the atmosphere. Thus, a contribution to the net enthalpy flux from the ocean to the atmosphere can be provided only by the fraction of spray droplets that fall back into the ocean. The sizes of the droplets that fall to the ocean can be estimated from the criterion that the velocity of their gravitational settling exceeds the wind friction velocity $u_{*}$. The estimates in the appendix show that spray droplets with radii above $150 \mu \mathrm{m}$ will fall down to the ocean even at hurricane winds. The integral relations derived by Andreas and Emanuel (2001) are not sufficient for evaluating the partial contributions of droplets of different sizes to the heat and enthalpy fluxes. To do this, we need to determine the fluxes released to the atmosphere from a single droplet.

\section{a. The enthalpy flux}

Here we derive an expression for the enthalpy flux and sensible and latent heat lost from the droplets to the atmosphere based on the droplet microphysics equations discussed by Pruppacher and Klett (1978). The equation for the evolution of the droplet temperature is derived from the condition that the total heat (or enthalpy) flux to the droplet (the integral of the heat flux density over the entire droplet surface) $Q_{\text {drop }}^{\text {total }}$ is equal to the sum of the diffusion heat flux due to the thermal conductivity of the atmosphere $F_{\text {atm } \rightarrow \text { drop }}$ and the latent heat flux due to the phase transition at the surface of the droplet $L_{v} d m / d t$.

Equating $Q_{\text {drop }}^{\text {total }}$ to the rate of change of the droplet heat content (or enthalpy) gives (Pruppacher and Klett 1978)

$$
C_{w} m \frac{d T}{d t}=F_{\text {atm } \rightarrow \text { drop }}+L_{v} \frac{d m}{d t},
$$

where $C_{w}$ is the specific heat of saltwater and $m$ and $T$ are the mass and absolute temperature of the droplet. 
On the other hand, a change in the moist enthalpy (the total heat content of the atmosphere) occurs as the droplet exchanges heat with the atmosphere through two physical processes. The first is the thermal conductivity, which provides the diffusion heat flux from the droplet to the atmosphere. This is opposite to the diffusion flux from the atmosphere to the droplet: $F_{\text {drop } \rightarrow \text { atm }}=-F_{\text {atm } \rightarrow \text { drop }}$. Another process is evaporation, which increases the internal energy of the atmosphere due to the water vapor flux and provides the latent heat flux equal to $-L_{v} d m / d t$.

Given Eq. (25), the enthalpy flux from the droplet to the atmosphere is evaluated as

$$
F_{k}=-F_{\text {atm } \rightarrow \text { drop }}-L_{v} \frac{d m}{d t}=-C_{w} m \frac{d T}{d t} .
$$

Equation (26), in particular, shows that in the stationary state, when equilibrium is established between the diffusion heat flux and the heat lost from the droplet due to its evaporation, the enthalpy flux from the droplet to the atmosphere ceases in agreement with Andreas and Emanuel (2001).

The amount of enthalpy entering the atmosphere over the entire life cycle of the droplet from its injection to its fall back into the ocean is then given by

$$
Q_{k}=\int_{0}^{\tau_{f}} F_{k} d t=-C_{w} \int_{0}^{\tau_{f}} m \frac{d T}{d t} d t .
$$

In Eq. (27), $\tau_{f}$ is the residence time of spray droplets in the air above the sea surface, which we estimate as follows:

$$
\tau_{f}=\frac{z_{s}}{V_{f}(r)},
$$

where $V_{f}(r)$ is the gravitational settling velocity of droplets and $z_{s}$ is the level of spray generation defined by Eq. (18) and proportional to the significant wave height. Notice that Eq. (28) is a crude estimate often used for the time of residence of the droplet in the atmosphere. A more accurate estimate requires sophisticated models, like the stochastic Lagrangian models (see Edson and Fairall 1994; Mueller and Veron 2014a; Troitskaya et al. 2016), which are also problematic due to uncertainty in the distribution of the ejection velocities most essential for the spume droplets.

The temporal development of the droplet temperature and mass necessary for evaluating the enthalpy of droplets in Eq. (27) was investigated by Andreas (1990, 1992, 1995, 2005) by employing the droplet microphysics equations formulated by Pruppacher and Klett (1978).

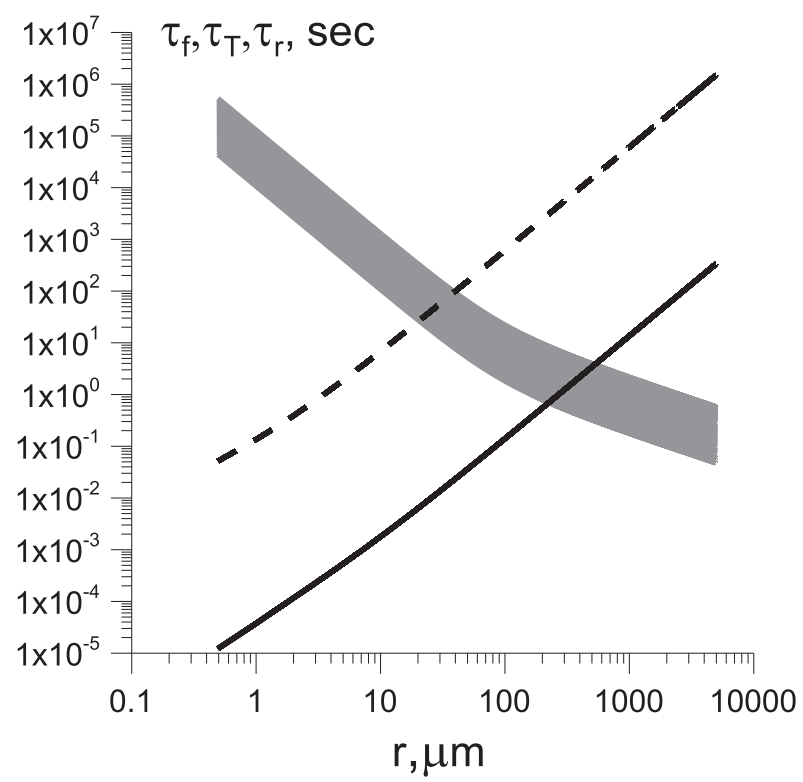

FIG. 4. Dependence of droplet evolution time scales vs radius $r$ : evaporation time $\tau_{r}$, (dashed curve), residence time in the air $\tau_{f}$ at $U_{10}$ between 30 and $60 \mathrm{~m} \mathrm{~s}^{-1}$ in $2 \mathrm{~m} \mathrm{~s}^{-1}$ increments (gray strip), and temperature relaxation time $\tau_{T}$ (solid curve). Bulk parameters are $U_{10}=30-60 \mathrm{~m} \mathrm{~s}^{-1}, T_{w}=25^{\circ} \mathrm{C}, T_{a}=27.5^{\circ} \mathrm{C}$, and $\mathrm{RH}=96 \%$; waveage parameter $\Omega=2.5$.

Approximate formulae for the droplet temperature $T(t)$ and radius $r(t)$ were obtained by Andreas (2005) in the following form:

$$
\begin{aligned}
& r(t)=r_{\text {eq }}+\left(r_{0}-r_{\text {eq }}\right) \exp \left(-t / \tau_{r}\right), \quad \text { and } \\
& T(t)=T_{\text {eq }}+\left(T_{w}-T_{\text {eq }}\right) \exp \left(-t / \tau_{T}\right) .
\end{aligned}
$$

In Eqs. (28)-(30), $T_{w}$ is the temperature of the sea surface; $T_{\text {eq }}$ is the droplet temperature at its thermal equilibrium, which is lower than $T_{w}$ due to spray evaporation; and $\tau_{T}$ and $\tau_{r}$ are the relaxation times of the droplet temperature and evaporation. The parameters in Eqs. (28)-(30) were evaluated with the use of software developed by Andreas (2013).

Figure 4 shows the dependence of droplet evolution time scales, $\tau_{r}, \tau_{f}$, and $\tau_{T}$, on the radius $r$. The figure shows that $\tau_{r} \gg \tau_{f}$ for large droplets with radius $r>$ $100 \mu \mathrm{m}$, that is, the size of these droplets essentially does not change before they fall back into the water. On the other hand, for these droplets $\tau_{T}$ is less or comparable to $\tau_{f}$, which means that the droplets have enough time to cool to their equilibrium temperature with an insignificant change in their size.

Approximate Eqs. (29) and (30) can be used to calculate the amount of enthalpy transferred to the atmosphere from a droplet with density $\rho_{w}$ : 


$$
\begin{aligned}
Q_{k}= & -\frac{4}{3} \pi \rho_{w} C_{w} \int_{0}^{\tau_{f}} r(t)^{3} \frac{d T(t)}{d t} d t=-\frac{4}{3} \pi \rho_{w} C_{w}\left(T_{w}-T_{\mathrm{eq}}\right) \exp \left(-t / \tau_{T}\right) \\
& \times\left[r_{\mathrm{eq}}^{3}+3 r_{\mathrm{eq}}^{2}\left(r_{0}-r_{\mathrm{eq}}\right) \frac{\tau_{r} \exp \left(-t / \tau_{r}\right)}{\tau_{T}+\tau_{r}}+3 r_{\mathrm{eq}}\left(r_{0}-r_{\mathrm{eq}}\right)^{2} \frac{\tau_{r} \exp \left(-2 t / \tau_{r}\right)}{2 \tau_{T}+\tau_{r}}+\left(r_{0}-r_{\mathrm{eq}}\right)^{3} \frac{\tau_{r} \exp \left(-3 t / \tau_{r}\right)}{3 \tau_{T}+\tau_{r}}\right]_{0}^{\tau_{f}} .
\end{aligned}
$$

Since $\tau_{T} / \tau_{r} \sim 10^{-3}$ (see Fig. 4), Eq. (31) can be simplified and, with the use of Eqs. (29) and (30), yields

$$
Q_{k}=C_{w}\left\{m_{0}\left(T_{w}-T_{\mathrm{eq}}\right)+m_{f}\left[T_{\mathrm{eq}}-T\left(\tau_{f}\right)\right]\right\}
$$

where $m_{0}=(4 / 3) \pi \rho_{w} r_{0}^{3} ; m_{f}=(4 / 3) \pi \rho_{w} r\left(\tau_{f}\right)^{3}$ is the droplet mass at the moments of its injection and reentrance into the water, respectively.

Equation (32) can also be derived from Eq. (27) without using approximate Eqs. (29) and (30), provided that the droplet evaporation time scale $\tau_{r}$ far exceeds the thermal relaxation time $\tau_{T}$. Suppose that the residence time of spray droplets in the air $\tau_{f}$ also significantly exceeds $\tau_{T}$. Then we can split the integral in Eq. (27) into two parts:

$$
\begin{aligned}
Q_{k}=-C_{w} \int_{0}^{\tau_{f}} m \frac{d T}{d t} d t= & -C_{w}\left(\int_{0}^{M \tau_{T}} m \frac{d T}{d t} d t\right. \\
& \left.+\int_{M \tau_{T}}^{\tau_{f}} m \frac{d T}{d t} d t\right),
\end{aligned}
$$

where $M>1$, so that $T(t)$ relaxes to equilibrium $T_{\text {eq }}$ by time $t=M \tau_{T}$. Since $\tau_{r} \gg \tau_{T}$, in the first integral in brackets we can put $m \approx m_{0}$, so that $I_{1}=\int_{0}^{M \tau_{T}} m(d T / d t) d t=m_{0}\left(T_{\text {eq }}-T_{w}\right)$. Evaluating the second integral in brackets by parts gives $I_{2}=\int_{M \tau_{T}}^{\tau_{f}} m(d T / d t) d t=\left.m T\right|_{M \tau_{T}} ^{\tau_{f}}-\int_{M \tau_{T}}^{\tau_{f}} T(d m / d t) d t$ with $T \approx T_{\text {eq }}$ in the integral in the latter part. Then $I_{2}=m_{f}\left[T\left(\tau_{f}\right)-T_{\text {eq }}\right]$, and finally the expression for $Q_{k}=$ $-C_{w}\left[I_{1}+I_{2}\right]$, coinciding with Eq. (32).

Figure 4 shows that $\tau_{T}$ is comparable with $\tau_{f}$ for droplets with radii larger than $200 \mu \mathrm{m}$; for these droplets, $\tau_{r} \gg \tau_{f}$ and $m_{f}=m_{0}$. Equation (32) can then be rewritten as

$$
Q_{k}=C_{w} m_{0}\left[T_{w}-T\left(\tau_{f}\right)\right] .
$$

For droplets where $\tau_{T} \ll \tau_{f}$, we obtain $T\left(\tau_{f}\right)=T_{\text {eq }}$, and Eq. (32) yields

$$
Q_{k}=C_{w} m_{0}\left(T_{w}-T_{\text {eq }}\right),
$$

which is a particular case of Eq. (33) for $T\left(\tau_{f}\right)=T_{\mathrm{eq}}$.

Note that Eq. (32) for the enthalpy transferred from one droplet to the atmosphere differs from the formulation of Andreas and Emanuel (2001), which was obtained from the conservation of the total enthalpy of the system [cf. Eq. (3) in their paper]. In terms of this paper,
Eq. (3) of Andreas and Emanuel (2001) can be rewritten in the form:

$$
Q_{k}^{A-E}=C_{w}\left\{m_{0}\left(T_{w}-T_{a}\right)+m_{f}\left[T_{a}-T\left(\tau_{f}\right)\right]\right\},
$$

where $T_{a}$ is the temperature of the atmosphere in close vicinity to the droplet. Note that both Eq. (32) and Eq. (34) give practically the same value for the transferred enthalpy since the dominant contribution to the enthalpy flux is from large droplets, the masses of which change insignificantly in the course of their life cycles, that is, $m_{0} \approx m_{f}$. For these droplets, both expressions are reduced to Eq. (33).

\section{b. The latent and sensible heat fluxes}

The latent heat taken from the atmosphere due to the evaporation of a given droplet equals

$$
Q_{L}=L_{v}\left(m_{0}-m_{f}\right),
$$

and the enthalpy released from this droplet to the atmosphere from ejection to reentry into the ocean is given by Eq. (32). Thus, the sensitive heat transferred to the atmosphere from this droplet due to the air thermal diffusivity is evaluated as

$$
\begin{aligned}
Q_{S}= & Q_{k}-Q_{L}=C_{W} m_{0}\left[T_{w}-T\left(\tau_{f}\right)\right] \\
& +\left(m_{0}-m_{f}\right)\left\{C_{W}\left[T\left(\tau_{f}\right)-T_{\mathrm{eq}}\right]-L_{v}\right\} .
\end{aligned}
$$

Equations (36) and (33) show that for large droplets with $\tau_{R} \gg \tau_{f}$ and $m_{f}=m_{0}$, the enthalpy released to the atmosphere is governed by the sensible heat transfer due to the thermal diffusivity of the air, similarly to the conclusion of Andreas and Emanuel (2001).

Using the SGF developed in Part I, we now evaluate the contribution of the spray generated by the bag breakup to the enthalpy and latent and sensible heat fluxes as follows:

$$
H_{X d}=\int_{0}^{\infty} \frac{d F\left(r, u_{*}\right)}{d r} Q_{X} d r
$$

where one-droplet contributions to the enthalpy and latent and sensible heat are denoted by $Q_{X}$ and given by Eqs. (32), (35), and (36), respectively.

Figures $5 \mathrm{a}$ and $5 \mathrm{c}$ show the partial contribution of droplets of different sizes to the enthalpy and latent and sensible heat fluxes from spray to atmosphere, namely, 
the integrand of the integral in Eq. (37), $d F\left(r, u_{*}\right) / d r Q_{X}$, for atmospheric parameters typical for tropical cyclones (Zhang et al. 2013) and two models of the fetch dependence of the SGF. The figure shows that the major contribution to the enthalpy flux is from large droplets with radii larger than $200 \mu \mathrm{m}$. The peak contribution from droplets with radii of $350-400 \mu \mathrm{m}$ is determined by the balance between the number of spray droplets with a certain radius ejected from the water surface defined by the $\mathrm{SGF}^{1}$ and the contribution of a single droplet determined by its mass and time of residence in the airflow according to Eq. (32). The figure also shows that the enthalpy flux released by these droplets is dominated by the sensible heat flux contribution (cf. solid and gray dashed curves). The share of latent heat flux is relatively insignificant since only a small fraction of these large droplets evaporates before they reenter the water (cf. Andreas and Emanuel 2001). Latent $E_{L d}$ and sensible $E_{S d}$ heat fluxes are maximal for the canopy droplets with radii of approximately $30 \mu \mathrm{m}$, though they have different signs: $E_{L d}>0$ and $E_{S d}<0$ (Figs. 5a,c). This confirms that the heat flux to droplets during their evaporation is mostly extracted from the atmosphere (cf. Hasse 1992; Emanuel 1995). For small droplets, the latent and sensible heat fluxes compensate for each other, so that they give practically no contribution to the net enthalpy flux. Figures $5 \mathrm{~b}$ and $5 \mathrm{~d}$ also show that under the conditions of very high relative humidity considered here, the contributions of the spray-mediated latent and sensible heat fluxes to the total enthalpy flux are comparable. Figures $5 \mathrm{~b}$ and $5 \mathrm{~d}$ show similar peculiarities in the spray-mediated heat fluxes and the momentum flux as seen in Figs. 2b and 2d, namely, that Eq. (8) predicts similar values and faster saturation at high winds and lower values at winds below $30 \mathrm{~m} \mathrm{~s}^{-1}$ than Eq. (2).

\section{Contribution of bag breakup to the air-sea enthalpy flux at hurricane wind speeds}

Let us now consider the contribution of spray to the exchange coefficient of moist enthalpy, which, along with the surface drag coefficient, is another key factor in the hurricane energy balance (Emanuel 1995).

The specific moist enthalpy is defined as a superposition of sensible $S=(1-q) c_{p} T+q c_{p v} T$ and latent $L=q L_{v}$ heat as

$$
k=(1-q) c_{p} T+q\left(L_{v}+c_{p v} T\right)
$$

\footnotetext{
${ }^{1}$ Note that SGFs are proportional to the value $P$ in Figs. 2 a and $2 \mathrm{c}$.
}

where $q$ and $c_{p}$ are the specific humidity and heat of air at constant pressure, $L_{v}$ is the latent heat of vaporization of water, $T$ is the air temperature, and $c_{p v}$ is the specific heat of water vapor at constant pressure.

The total enthalpy flux from the ocean to the atmosphere consists of two components: the direct turbulent interfacial flux $H_{k t}$ and the spray-mediated flux $H_{k d}$. The turbulent flux $H_{k t}$ is usually expressed via bulk formula as

$$
H_{k t}=C_{k 0} U_{10}\left(k_{10}-k_{s}\right)
$$

where $C_{k 0}$ is an empirical enthalpy transfer coefficient. This coefficient is practically independent of the wind speed at winds below $30 \mathrm{~m} \mathrm{~s}^{-1}$ (e.g., Black et al. 2007) and equals $0.0011-0.0012$. Parameters $k_{10}$ and $k_{s}$ are the values of specific moist enthalpy at the reference height, $10 \mathrm{~m}$, and at the water surface, respectively. The spray-mediated flux $H_{k d}$ is defined by Eq. (36) for $X=k$. The contribution of droplets of a certain radius $r$, $d F\left(r, u_{*}\right) / d r Q_{k}$, to the enthalpy flux is shown in Fig. 6a. The figure confirms the dominant contribution from giant rim droplets to the spray-mediated enthalpy flux, especially at winds exceeding $35 \mathrm{~m} \mathrm{~s}^{-1}$ for both models of the fetch dependence of the SGF.

The total enthalpy flux is given by

$$
H_{k}=H_{k t}+H_{k d} .
$$

Figure $6 \mathrm{~b}$ presents the dependence of the spraymediated enthalpy flux $H_{k d}$ and direct turbulent enthalpy transfer $H_{k t}$ on the wind speed. The figure shows that at winds exceeding $30 \mathrm{~m} \mathrm{~s}^{-1}$, both models give very similar values for the spray-mediated enthalpy flux. A pronounced difference occurs only at wind speeds below $30 \mathrm{~m} \mathrm{~s}^{-1}$, where the Eq. (2) model predicts more bag-breakup events at fetches typical of field conditions. Figure $6 \mathrm{~b}$ also shows that at wind speeds above $45 \mathrm{~m} \mathrm{~s}^{-1}$, when the spray-mediated flux is determined mostly by the giant rim droplets, the spraymediated flux exceeds the turbulent enthalpy flux. Figure $6 \mathrm{c}$ shows that both models predict more than $50 \%$ of the total enthalpy flux to the atmosphere mediated by spray at winds above $40-50 \mathrm{~m} \mathrm{~s}^{-1}$.

The enthalpy exchange coefficient for the neutrally stable atmospheric boundary layer is, by definition,

$$
C_{k N}=H_{k} /\left[\rho_{a} U_{10}\left(k_{10}-k_{s}\right)\right] .
$$

The effect of the stable stratification of the marine atmospheric boundary layer due to suspended droplets on the enthalpy exchange coefficient can be taken into account in a similar manner to the drag coefficient [cf. Eq. (24)] as 


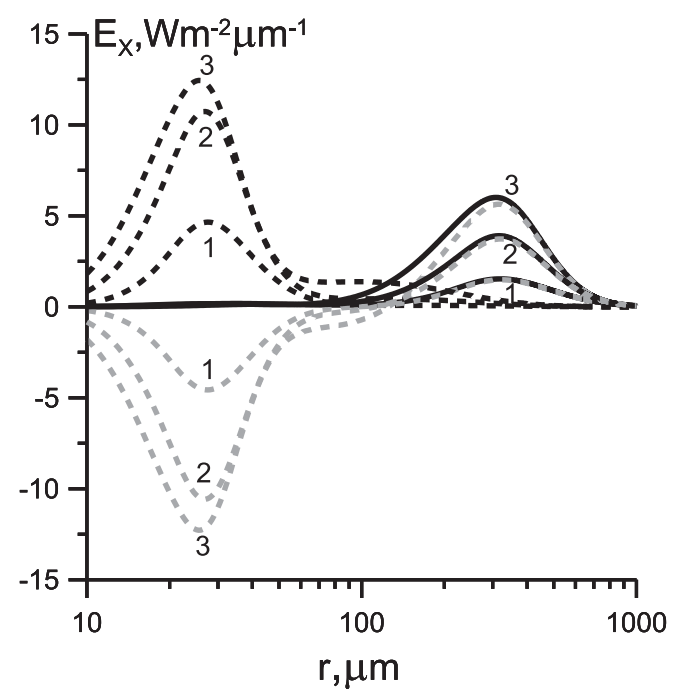

(a)

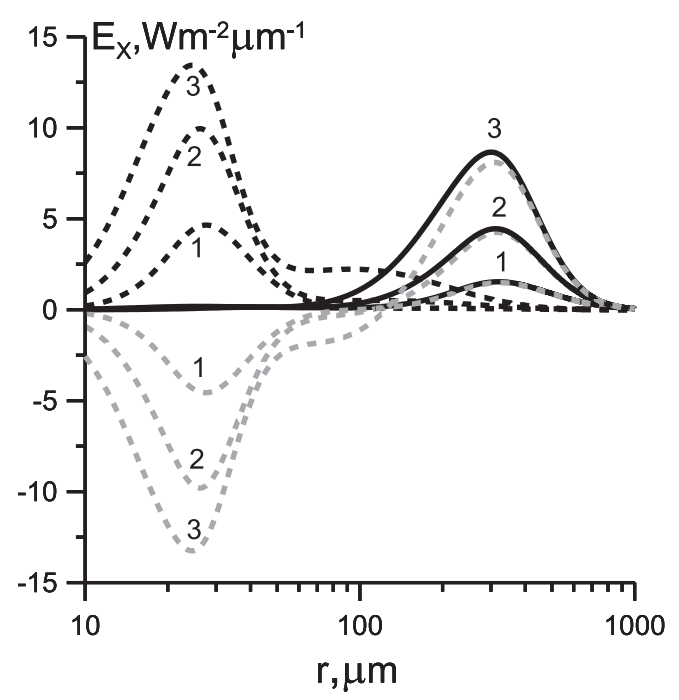

(c)

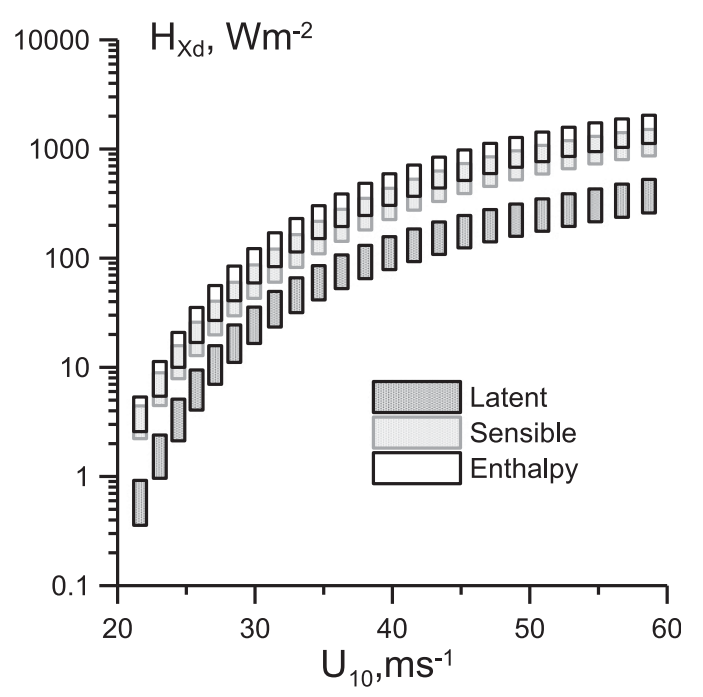

(b)

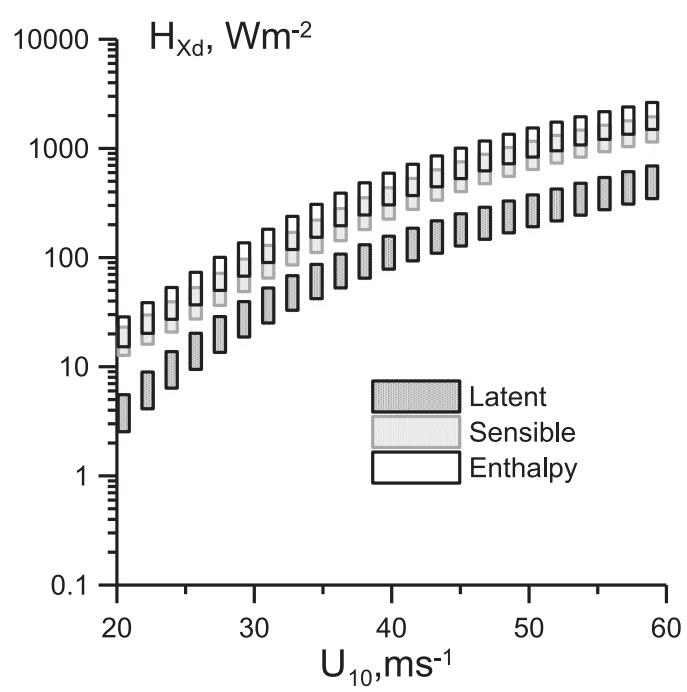

(d)

FIG. 5. Contribution of the droplets originating from bag breakup to air-sea heat exchange: calculations use (top) Eq. (8) and (bottom) Eq. (2) for the specific number of bags. (a),(c) Partial contribution of droplets to the total enthalpy flux (solid curve) and to latent (dashed black curve) and sensible (dashed gray curve) heat fluxes. Values of $U_{10}$ are $40 \mathrm{~m} \mathrm{~s}^{-1}$ (marker 1), $50 \mathrm{~m} \mathrm{~s}^{-1}$ (marker 2), and $60 \mathrm{~m} \mathrm{~s}^{-1}$ (marker 3); the wave-age parameter $\Omega=2.5, T_{w}=$ $27.5^{\circ} \mathrm{C}, T_{a}=25^{\circ} \mathrm{C}$, and $\mathrm{RH}=96 \%$. (b),(d) Spray-mediated latent and sensible heat and the total enthalpy fluxes for the same thermodynamic parameters of the atmosphere as in (a) and (c); the vertical bars depict variations in the wave-age parameter $\Omega$ from 2.5 to 3.5 .

$$
C_{k}=\frac{C_{k N}}{\left[1+\beta \frac{\rho_{w} g}{\rho_{a} u_{*}^{2}} \sqrt{C_{D N}} \int_{z_{s}}^{\infty} d z_{1} \int V_{f}(r) S\left(z_{1}, r\right) d r\right]^{2}} .
$$

The dependencies of the enthalpy transfer coefficient $C_{k}$ on the wind speed are shown in Fig. 7a. Both models predict similar values of $C_{k}$ within the uncertainty caused the by variation in wave-age parameter $\Omega$. The growth of
$C_{k}$ with wind exceeding $35-40 \mathrm{~m} \mathrm{~s}^{-1}$ at $U_{10}$ results from the increase in the spray-mediated flux at these wind speeds (cf. with Fig. 6b). The predicted values of $C_{k}$ differ only at winds above $50 \mathrm{~m} \mathrm{~s}^{-1}$ and then by no more than $8 \%-10 \%$. Consequently, the ratio of the exchange coefficients $C_{k} / C_{D}$ increases and, at $35-40 \mathrm{~m} \mathrm{~s}^{-1}$ wind, exceeds 0.75 , the lower limit for realistic model prediction of the maximum wind speed a hurricane can attain at the 


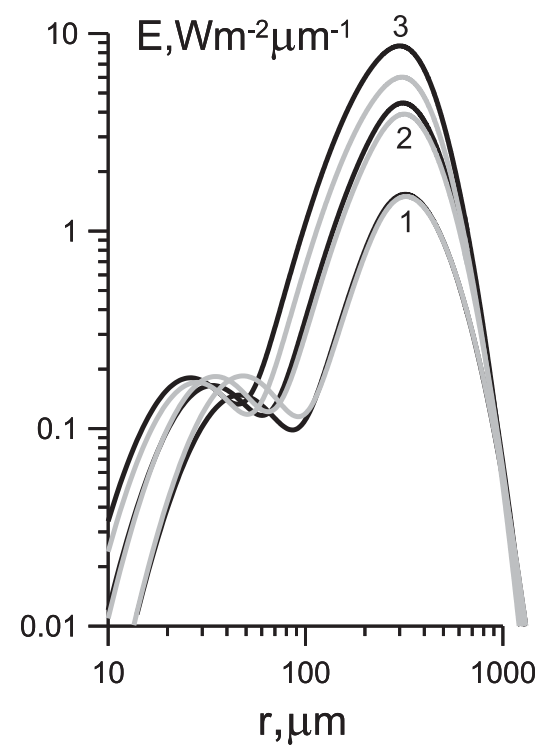

(a)

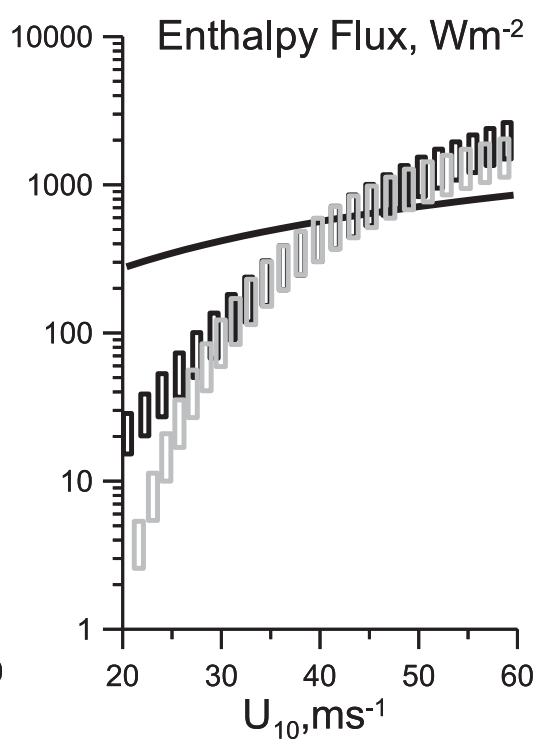

(b)

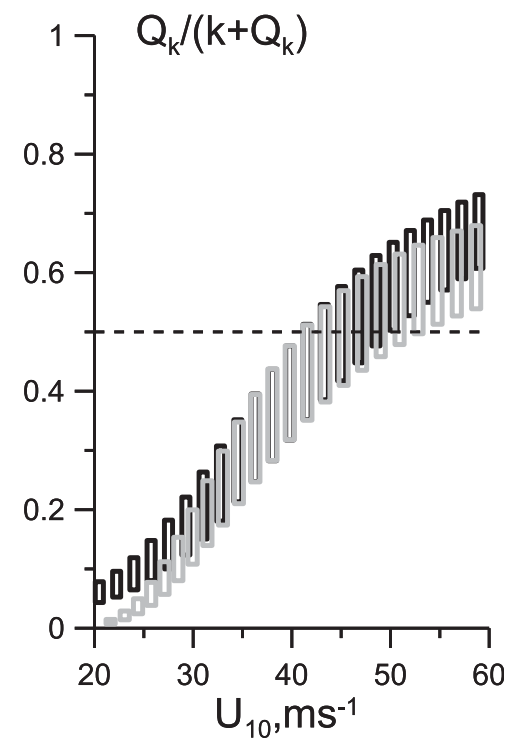

(c)

FIG. 6. (a) Partial contributions of droplets to the enthalpy flux $E$. Values of $U_{10}$ are $40 \mathrm{~m} \mathrm{~s}^{-1}$ (marker 1), $50 \mathrm{~m} \mathrm{~s}^{-1}$ (marker 2), and $60 \mathrm{~m} \mathrm{~s}^{-1}$ (marker 3); the wave-age parameter $\Omega=2.5, T_{w}=27.5^{\circ} \mathrm{C}, T_{a}=25^{\circ} \mathrm{C}$, and $\mathrm{RH}=96 \%$. (b) Contributions to the enthalpy flux from droplets (floating bars) and from direct turbulent transfer (black solid curve). (c) Fractional contribution of spray from bag breakup to the enthalpy flux. The dashed line denotes 0.5 . The gray lines correspond to calculations using Eq. (8), and the black lines correspond to those using Eq. (2) for the specific number of bags; the vertical bars in (b) and (c) depict variations in the wave-age parameter $\Omega$ from 2.5 to 3.5 . The thermodynamic parameters of the atmosphere are the same as in (a).

observed parameters of the atmosphere and ocean as discussed by Emanuel (1995) (Fig. 7b).

It should be taken into account that the above estimates give only the upper limits of the enthalpy flux and exchange coefficient since they neglect the feedback effects of droplets on the temperature and moisture profiles of the marine atmospheric boundary layer. These feedback effects can substantially reduce the contribution of droplets, as discussed by Bao et al. $(2000,2011)$, Bianco et al. (2011), and Mueller and Veron (2014b). However, a number of uncertainties complicate precise estimates of the feedback effect of spray. The most significant is the uncertainty in the initial velocities of droplets ejected from the water surface, which strongly influence the spray-mediated momentum flux [see discussion in Troitskaya et al. (2016) and Druzhinin et al. (2017)].

\section{Summary}

In Part I of this study, we showed that the dominant generation mechanism of spume droplets (i.e., drops of spray torn from the crests of waves by the wind) is that of bag-breakup fragmentation. This new spray-production mechanism is characterized by the formation of specific short-living (a lifetime of circa $10 \mu \mathrm{s}$ ) objects, bags, which look like canopies with radii of $\sim 1-2 \mathrm{~cm}$ supported by thicker liquid rims. This structure results in the production of two scales of droplets after bag bursting and a doublepeaked size spectrum of droplets with maxima at $\sim 100 \mu \mathrm{m}$ (the "film" droplets) and 500-1000 $\mu \mathrm{m}$ (the giant "rim" droplets). The bags and the giant droplets, the distinctive feature of the bag-breakup mechanism, turn out to make a significant contribution to both the heat energy supply from the ocean and mechanical dissipation in the atmospheric boundary layer, that is, factors responsible for the development and maintenance of hurricanes (Emanuel 1995).

In particular, the effect of bags and giant droplets can explain the nonmonotonous dependence of the surface drag coefficient $C_{D}$ on wind speed seen in meteorological (Powell et al. 2003; Holthuijsen et al. 2012) and oceanographic (Jarosz et al. 2007) observations. Indeed, the contribution of bag breakup to the momentum flux consists of two parts:

- the droplet stress, equal to the amount of momentum acquired by droplets in the course of their production, and

- the bag stress, provided by bags acting as microsails with typical sizes of $\sim 1 \mathrm{~cm}$, which act as obstacles to the near-water airflow.

Bag stress as well as droplet stress levels off at high winds due to the competition between two counteracting 


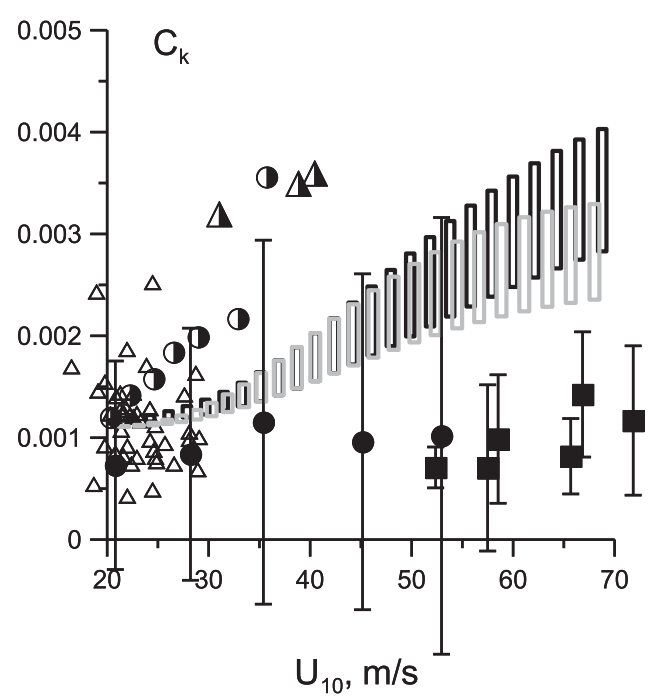

(a)

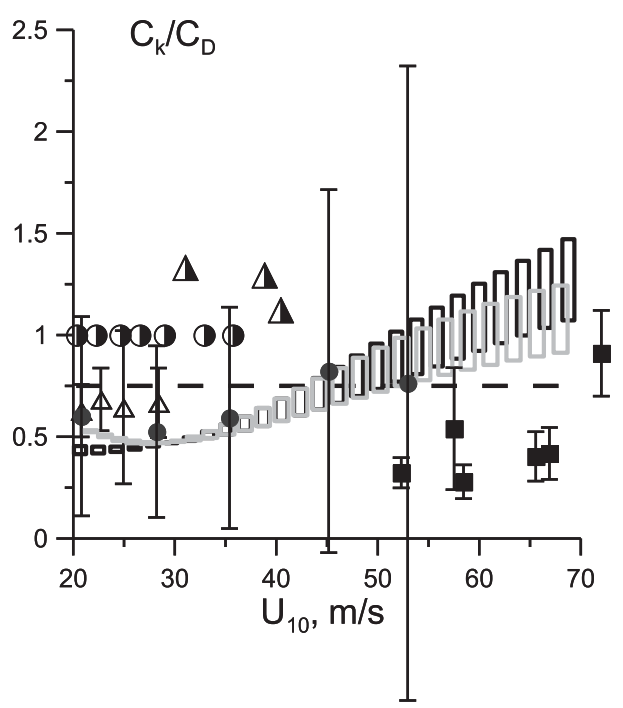

(b)

FIG. 7. (a) The enthalpy transfer coefficient $C_{k}$ vs $U_{10}$. Experimental data: open triangles are from Black et al. (2007), circles are from Richter et al. (2016), squares are from Bell et al. (2012), half-closed circles are from Hawkins and Rubsam (1968), and half-closed triangles are from Miller (1962). (b) The ratio of the enthalpy and drag coefficients vs $U_{10}$. The dashed line denotes the level 0.75. Experimental data: open triangles are from Black et al. (2007), closed circles are from Richter et al. (2016), squares are from Bell et al. (2012), half-closed circles are from Hawkins and Rubsam (1968), and half-closed triangles are from Miller (1962). The vertical bars depict variations in $\Omega$ from 2.5 to $3.5, T_{w}=27.5^{\circ} \mathrm{C}, T_{a}=25^{\circ} \mathrm{C}$, and $\mathrm{RH}=96 \%$. The gray lines show calculations using Eq. (8), and the black lines show calculations using Eq. (2) for the specific number of bags.

effects of the increasing wind speed, namely, the increasing number of bags and droplets and the weakening individual contributions of bags and droplets to the momentum flux due to decreasing bag sizes and lifetimes. It should be emphasized that even though bags and spray increase the surface drag in comparison with the pure form drag of surface waves, the tendency to saturation of the spray-and-bag-mediated component of the surface stress has the potential to explain the nonmonotonous dependence of the surface drag coefficient on wind speed. We also estimated the effect of stable stratification of the near-surface airflow by suspended droplets, which is known to reduce the intensity of air turbulence in the marine atmospheric boundary layer (Kudryavtsev 2006; Bao et al. 2011). The effect is not strong, leading to a less than $8 \%$ reduction of the surface drag coefficient. However, a combination of the above three factors leads to a nonmonotonous dependence of the drag coefficient on the wind speed.

We adapted the concept of reentrant spray initially formulated by Andreas and Emanuel (2001), who considered a column of adjacent layers of the atmosphere and the ocean, assumed conservation of the total enthalpy, and employed microphysical equations describing the evolution of a single droplet derived by Pruppacher and Klett (1978). This concept indicates that the contribution from spray to the thermal energy supply from ocean to atmosphere (quantified by the vertical flux of moist enthalpy $H_{k}$ ) is provided by droplets large enough to cool down below the ambient air temperature (due to the evaporation of only a small fraction of their volume) and then reenter the water. Our estimates show that the dominant contribution to the spray-mediated enthalpy flux is from giant rim droplets, and especially so at winds exceeding $35 \mathrm{~m} \mathrm{~s}^{-1}$. The contribution of the giant reentrant droplets generated by bag breakup increases with an increase in the wind speed and finally exceeds the near-surface turbulent heat flux when the wind speed is above 45$50 \mathrm{~m} \mathrm{~s}^{-1}$. As a result, the enthalpy exchange coefficient increases with strengthening wind. Consequently, the ratio of the exchange coefficients $\mathrm{C}_{k} / \mathrm{C}_{D}$ increases and, at $35-40 \mathrm{~m} \mathrm{~s}^{-1}$ winds, exceeds 0.75 , the threshold predicted by Emanuel (1995) to reproduce the realistic intensity of tropical cyclones.

The above results show the critical importance of taking into account the contribution of large droplets and bags to the heat and momentum atmosphereocean exchange at high winds. However, these estimates 
are rather crude and give only upper limits to the enthalpy flux and exchange coefficient. Obtaining realistic quantitative estimates requires consideration of the feedback effect of the spray-mediated fluxes, which induce perturbations of the temperature and moisture profiles and reduce the spray-mediated fluxes (Bao et al. 2011; Bianco et al. 2011; Mueller and Veron 2014b). At the moment, the principal obstacle to solving this problem in a consistent way is uncertainty regarding droplet velocities at injection from the ocean surface into the atmosphere during bag breakup. Recent direct numerical simulation results show that the variation of droplet velocities at injection strongly affects the spraymediated momentum flux (Druzhinin et al. 2017).

One other problem for the realistic estimation of the effect of bag-breakup fragmentation on air-sea fluxes is how to model the fetch dependence of the number of bagbreakup events, which determines the SGFs. Here we have compared two empirical models based on laboratory tank data and parameterization in terms of the windsea Reynolds number introduced by Toba and Koga (1986). At the moment, the available data do not allow a definitive choice to be made between the discussed models, but Eq. (2) looks more promising as it predicts realistic essential spray production at winds of $10-15 \mathrm{~m} \mathrm{~s}^{-1}$ at fetches typical for open-ocean conditions.

Acknowledgments. This work has been supported by the Russian Science Foundation (Project 14-1700667), the Seventh Framework Programme (Project PIRSES-GA-2013-612610), and the Russian Foundation for Basic Research (Projects 14-17-00667, 16-05-00839, 17-05-00703, 18-55-50005). Sergej Zilitinkevich additionally acknowledges support from the Academy of Finland Project ClimEco 314 798/799. The basic salary of the authors from IAP is financed by the government contract 0035-2014-0032. The experiments were performed at the Unique Scientific Facility "Complex of Large-Scale Geophysical Facilities” (http://www.ckp-rf.ru/usu/77738/) and were supported by the President Grant for Young Scientists MC-2041.2017.5 and Russian Science Foundation (Project 18-77-00074). The authors thank Ms. Rebecca Thompson for improving the use of English in and organization of the text.

\section{APPENDIX}

\section{Estimates of Giant Droplet Parameters in Terms of Exchange Processes}

First, we estimate the ratio of the relaxation time required for a droplet to adjust to the velocity of the surrounding airflow $\tau_{\text {Stokes }}$ and the time of residence in the atmosphere $\tau_{f}$ for a droplet ejected to the airflow from a height of $z_{s}$. Consider the droplet as a small sphere of radius $r$ under the forcing of viscous resistance and gravity and suppose that interaction between droplets is neglected. The momentum exchange of the droplet with the turbulent airflow is then described as

$$
\frac{4}{3} \pi \rho_{w} r^{3} \frac{d \mathbf{v}_{d}}{d t}=6 \pi \rho_{a} \nu_{a} r K(\operatorname{Re})\left(\mathbf{v}_{d}-\mathbf{U}\right)+\frac{4}{3} \pi \rho_{w} r^{3} \mathbf{g} .
$$

Here, $\mathbf{v}_{d}$ is the droplet velocity, $\mathbf{U}$ is the velocity of the surrounding air, $\mathbf{g}$ is the vector of gravitational acceleration, $\nu_{a}$ is the kinematic viscosity of the air, $\rho_{a}$ and $\rho_{w}$ are the air and droplet density, $K(\mathrm{Re})$ is a dimensionless parameter equal to the ratio of the resistance force at the droplet for the finite Reynolds number Re to the Stokes force. We used the function of $K(\mathrm{Re})$ from Andreas (1990):

$$
K(\mathrm{Re})=1+0.158 \mathrm{Re}^{2 / 3} ; \quad \mathrm{Re}=\frac{2 r V_{f}}{\nu_{a}} .
$$

The time $\tau_{\text {Stokes }}$ is determined as the relaxation time of the Stokes sphere modified for the finite Re:

$$
\tau_{\text {Stokes }}=\frac{2 \rho_{w}}{9 \rho_{a}} \frac{r^{2}}{\nu_{a} K(\mathrm{Re})} .
$$

The time of residence of the droplet in the atmosphere $\tau_{f}$ is determined by Eq. (28), where $V_{f}(r)$, the gravitational settling velocity of droplets, follows from Eq. (A1) as follows:

$$
V_{f}=\frac{2}{9} \frac{\rho_{w}}{\rho_{a}} \frac{r^{2} g}{\nu_{a} K(\mathrm{Re})} .
$$

Using Eqs. (A2), (A3), and (28) then gives for the ratio $\tau_{f} / \tau_{\text {Stokes }}=z_{s} g / V_{f}^{2}$. Given Eq. (18), this readily yields $\tau_{f} / \tau_{\text {Stokes }}=0.011 U_{10}^{2} / V_{f}^{2}$. It is clear from Fig. A1 a that the residence time in the atmosphere is less than the time for Stokes relaxation of spray droplets with radii above $150 \mu \mathrm{m}$ for a wind speed of $20 \mathrm{~m} \mathrm{~s}^{-1}$, above $300 \mu \mathrm{m}$ for a wind speed of $40 \mathrm{~m} \mathrm{~s}^{-1}$, and above $450 \mu \mathrm{m}$ for a wind speed of $60 \mathrm{~m} \mathrm{~s}^{-1}$.

Second, we estimate the sizes of droplets that will fall back into the ocean at high winds, that is, will not be suspended due to the effect of turbulent velocity fluctuations. The sizes of these droplets are estimated on the basis of the criterion that the velocity of their gravitational settling $V_{f}$, determined by Eq. (A3), exceeds the wind friction velocity $u_{*}$. For hurricane winds, $u_{*}$ is around $2 \mathrm{~m} \mathrm{~s}^{-1}$ according to Powell et al. (2003). Figure A1b shows that droplets with radii above $150 \mu \mathrm{m}$ will fall. 


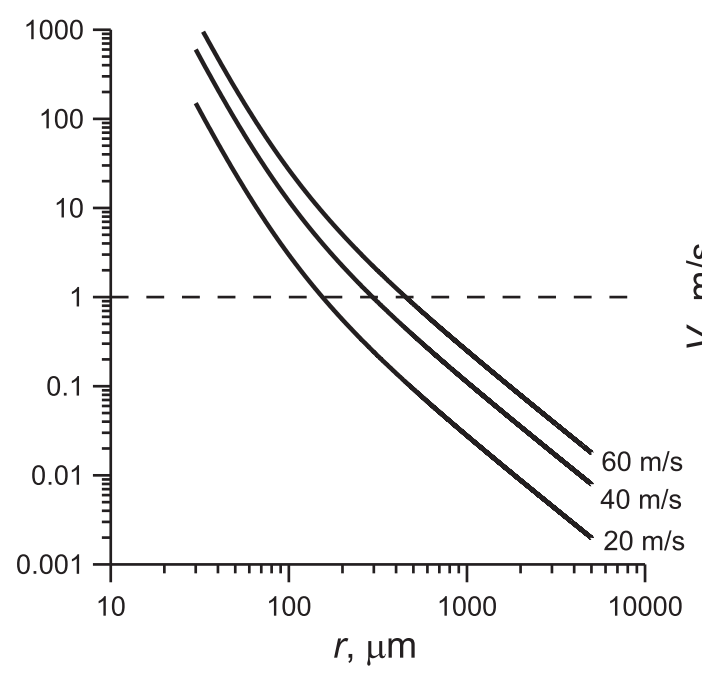

(a)

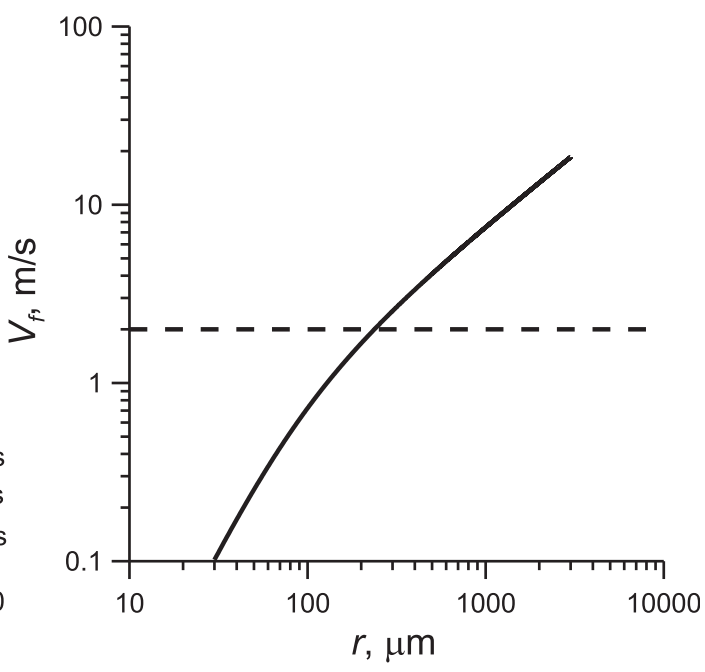

(b)

FIG. A1. (a) The ratio of the relaxation time for a sphere with $\tau_{\text {Stokes }}$ and time of residence of the droplet in the atmosphere vs $r$ for three values of wind speed; the dashed line marks 1. (b) The gravitational settling velocity of a droplet vs its radius; the dashed line corresponds to $2 \mathrm{~m} \mathrm{~s}^{-1}$.

\section{REFERENCES}

Andreas, E. L., 1990: Time constants for the evolution of sea spray droplets. Tellus, 42B, 481-497, https://doi.org/10.3402/ tellusb.v42i5.15241.

1992: Sea spray and the turbulent air-sea heat fluxes. J. Geophys. Res., 97, $11429-11441$, https://doi.org/10.1029/ 92JC00876.

_- 1995: The temperature of evaporating sea spray droplets. J. Atmos. Sci., 52, 852-862, https://doi.org/10.1175/15200469(1995)052<0852:TTOESS $>2.0$.CO;2.

_ 1998: A new sea spray generation function for wind speeds up to $32 \mathrm{~m} \mathrm{~s}^{-1}$. J. Phys. Oceanogr., 28, 2175-2184, https://doi.org/ 10.1175/1520-0485(1998)028<2175:ANSSGF>2.0.CO;2.

- 2002: A review of the sea spray generation function for the open ocean. Atmosphere-Ocean Interactions, Vol. 1, W. Perrie, Ed., WIT Press, 1-46.

, 2004: Spray stress revisited. J. Phys. Oceanogr., 34, 1429-1440, https://doi.org/10.1175/1520-0485(2004)034<1429:SSR > 2.0.CO;2.

— 2005: Approximation formulas for the microphysical properties of saline droplets. Atmos. Res., 75, 323-345, https:// doi.org/10.1016/j.atmosres.2005.02.001.

_ 2013: An algorithm for fast microphysical calculations that predict the evolution of saline droplets. NorthWest Research Associates, https://people.nwra.com/resumes/andreas/software.php.

—_, and K. A. Emanuel, 2001: Effects of sea spray on tropical cyclone intensity. J. Atmos. Sci., 58, 3741-3751, https://doi.org/ 10.1175/1520-0469(2001)058<3741:EOSSOT >2.0.CO;2.

_ J. B. Edson, E. C. Monahan, M. P. Rouault, and S. D. Smith, 1995: The spray contribution to net evaporation from the sea: A review of recent progress. Bound.-Layer Meteor., 72, 3-52, https://doi.org/10.1007/BF00712389.

Bao, J.-W., J. Wilczak, J. Choi, and L. Kantha, 2000: Numerical simulations of air-sea interaction under high wind conditions using a coupled model: A study of hurricane development. Mon. Wea. Rev., 128, 2190-2210, https://doi.org/10.1175/15200493(2000)128<2190:NSOASI >2.0.CO;2.
— C. W. Fairall, S. A. Michelson, and L. Bianco, 2011: Parameterizations of sea-spray impact on the air-sea momentum and heat fluxes. Mon. Wea. Rev., 139, 3781-3797, https://doi.org/ 10.1175/MWR-D-11-00007.1.

Barenblatt, G., and G. Golitsyn, 1974: Local structure of mature dust storms. J. Atmos. Sci., 31, 1917-1933, https://doi.org/ 10.1175/1520-0469(1974)031<1917:LSOMDS>2.0.CO;2.

Bell, M. M., M. T. Montgomery, and K. A. Emanuel, 2012: Air-sea enthalpy and momentum exchange at major hurricane wind speeds observed during CBLAST. J. Atmos. Sci., 69, 31973222, https://doi.org/10.1175/JAS-D-11-0276.1.

Bianco, L., J.-W. Bao, C. W. Fairall, and S. A. Michelson, 2011: Impact of sea-spray on the atmospheric surface layer. Bound.-Layer Meteor., 140, 361-381, https://doi.org/10.1007/ s10546-011-9617-1.

Black, P. G., and Coauthors, 2007: Air-sea exchange in hurricanes: Synthesis of observations from the Coupled Boundary Layer Air-Sea Transfer experiment. Bull. Amer. Meteor. Soc., 88, 357-374, https://doi.org/10.1175/BAMS-88-3-357.

Borisenkov, E. P., 1974: Some mechanisms of atmosphere-ocean interaction under stormy weather conditions. Probl. Arct. Antarct., 43 (44), 73-83.

Bortkovskii, R. S., 1973: On the mechanism of interaction between the ocean and the atmosphere during a storm. Fluid Mech. Sov. Res., 2, 87-94.

Chou, W.-H., and G. M. Faeth, 1998: Temporal properties of secondary drop breakup in the bag breakup regime. Int. J. Multiphase Flow, 24, 889-912, https://doi.org/10.1016/S03019322(98)00015-9.

Druzhinin, O. A., Yu. I. Troitskaya, and S. S. Zilitinkevich, 2017: The study of droplet-laden turbulent airflow over waved water surface by direct numerical simulation. J. Geophys. Res. Oceans, 122, 1789-1807, https://doi.org/10.1002/2016JC012134.

Dyer, A. J., 1974: A review of flux-profile relationships. Bound.-Layer Meteor., 7, 363-372, https://doi.org/10.1007/BF00240838.

Edson, J. B., and C. W. Fairall, 1994: Spray droplet modeling: 1. Lagrangian model simulation of the turbulent transport of 
evaporating droplets. J. Geophys. Res., 99, 25 295-25311, https://doi.org/10.1029/94JC01883.

Emanuel, K. A., 1995: Sensitivity of tropical cyclones to surface exchange coefficients and a revised steady-state model incorporating eye dynamics. J. Atmos. Sci., 52, 3969-3976, https:// doi.org/10.1175/1520-0469(1995)052<3969:SOTCTS >2.0.CO;2.

Fairall, C. W., J. D. Kepert, and G. J. Holland, 1994: The effect of sea spray on surface energy transports over the ocean. The Global Atmosphere and Ocean System, Vol. 2, K. Katsaros, Ed., Gordon and Breach Science Publishers, 121-142.

Foreman, R. J., and S. Emeis, 2010: Revisiting the definition of the drag coefficient in the marine atmospheric boundary layer. J. Phys. Oceanogr., 40, 2325-2332, https://doi.org/10.1175/2010JPO4420.1.

Gelfand, B. E., 1996: Droplet breakup phenomena in flows with velocity lag. Prog. Energy Combust. Sci., 22, 201-265, https:// doi.org/10.1016/S0360-1285(96)00005-6.

Hasse, L., 1992: On the contribution of spray droplets to evaporation. Bound.-Layer Meteor., 61, 309-313, https://doi.org/ 10.1007/BF02042938.

Hawkins, H. F., and D. T. Rubsam, 1968: Hurricane Hilda, 1964: II. Structure and budgets of the hurricane on October 1 , 1964. Mon. Wea. Rev., 96, 617-636, https://doi.org/10.1175/ 1520-0493(1968)096<0617:HH >2.0.CO;2.

Holthuijsen, L. H., M. D. Powell, and J. D. Pietrzak, 2012: Wind and waves in extreme hurricanes. J. Geophys. Res., 117, C09003, https://doi.org/10.1029/2012JC007983.

Iida, N., Y. Toba, and M. Chaen, 1992: A new expression for the production rate of sea water droplets on the sea surface. J. Oceanogr., 48, 439-460, https://doi.org/10.1007/BF02234020.

Jarosz, E., D. A. Mitchell, D. W. Wang, and W. J. Teague, 2007: Bottom-up determination of air-sea momentum exchange under a major tropical cyclone. Science, 315, 1707-1709, https://doi.org/10.1126/science.1136466.

Kudryavtsev, V. N., 2006: On the effect of sea drops on the atmospheric boundary layer. J. Geophys. Res., 111, C07020, https://doi.org/10.1029/2005JC002970.

_- , and V. K. Makin, 2011: Impact of ocean spray on the dynamics of the marine atmospheric boundary layer. Bound.-Layer Meteor., 140, 383-410, https://doi.org/10.1007/ s10546-011-9624-2.

Lhuissier, H., and E. Villermaux, 2012: Bursting bubble aerosols. J. Fluid Mech., 696, 5-44, https://doi.org/10.1017/jfm.2011.418.

Ling, S. C., and T. W. Kao, 1976: Parameterization of the moisture and heat transfer process over the ocean under whitecap sea states. J. Phys. Oceanogr., 6, 306-315, https://doi.org/10.1175/ 1520-0485(1976)006<0306:POTMAH >2.0.CO;2.

Makin, V. K., 2005: A note on drag of the sea surface at hurricane winds. Bound.-Layer Meteor., 115, 169-176, https://doi.org/ 10.1007/s10546-004-3647-x.

Marmottant, P., and E. Villermaux, 2004: On spray formation. J. Fluid Mech., 498, 73-111, https://doi.org/10.1017/ S0022112003006529.

Miller, B. I., 1962: On the momentum and energy balance of Hurricane Helene (1958). National Hurricane Research Project Rep. 53, U.S. Weather Bureau, 19 pp., ftp://ftp.library. noaa.gov/noaa_documents.lib/NOAA_historic_documents/ WB/National_Hurricane_Research_Project_Report/NHRP_53_ 1962.pdf.

_ 1964: A study of the filling of Hurricane Donna (1960) over land. Mon. Wea. Rev., 92, 389-406, https://doi.org/10.1175/ 1520-0493(1964)092<0389:ASOTFO>2.3.CO;2.

Mueller, J. A., and F. Veron, 2014a: Impact of sea spray on air-sea fluxes. Part I: Results from stochastic simulations of sea spray drops over the ocean. J. Phys. Oceanogr., 44, 2817-2834, https://doi.org/10.1175/JPO-D-13-0245.1.

_, and — 2014b: Impact of sea spray on air-sea fluxes. Part II: Feedback effects. J. Phys. Oceanogr., 44, 2835-2853, https:// doi.org/10.1175/JPO-D-13-0246.1.

Powell, M. D., P. J. Vickery, and T. A. Reinhold, 2003: Reduced drag coefficient for high wind speeds in tropical cyclones. Nature, 422, 279-283, https://doi.org/10.1038/nature01481.

Pruppacher, H. R., and J. D. Klett, 1978: Microphysics of Clouds and Precipitation. D. Riedel, 714 pp.

Richter, D. H., R. Bohac, and D. P. Stern, 2016: An assessment of the flux profile method for determining air-sea momentum and enthalpy fluxes from dropsonde data in tropical cyclones. J. Atmos. Sci., 73, 2665-2682, https://doi.org/10.1175/JAS-D-15-0331.1.

Rouault, M. P., P. G. Mestayer, and R. Schiestel, 1991: A model of evaporating spray droplet dispersion. J. Geophys. Res., 96, 7181-7200, https://doi.org/10.1029/90JC02569.

Soloviev, A. V., R. Lukas, M. Donelan, B. K. Haus, and I. Ginis, 2014: The air-sea interface and surface stress under tropical cyclones. Sci. Rep., 4, 5306, https://doi.org/10.1038/srep05306.

Takagaki, N., S. Komori, N. Suzuki, K. Iwano, T. Kuramoto, S. Shimada, R. Kurose, and K. Takahashi, 2012: Strong correlation between the drag coefficient and the shape of the wind sea spectrum over a broad range of wind speeds. Geophys. Res. Lett., 39, L23604, https://doi.org/10.1029/2012GL053988.

$-, \ldots,-\ldots, \ldots$, and R. Kurose, 2016: Mechanism of drag coefficient saturation at strong wind speeds. Geophys. Res. Lett., 43, 9829-9835, https://doi.org/10.1002/2016GL070666.

Toba, Y., and M. Koga, 1986: A parameter describing overall conditions of wave breaking, whitecapping, sea-spray production and wind stress. Oceanic Whitecaps, E. C. Monahan and G. MacNiocaill, Eds., D. Reidel, 37-47.

Troitskaya, Yu., E. Ezhova, I. Soustova, and S. Zilitinkevich, 2016: On the effect of sea spray on the aerodynamic surface drag under severe winds. Ocean Dyn., 66, 659-669, https://doi.org/ 10.1007/s10236-016-0948-9.

A. Kandaurov, O. Ermakova, D. Kozlov, D. Sergeev, and S. Zilitinkevich, 2017: Bag-breakup fragmentation as the dominant mechanism of sea-spray production in high winds. Sci. Rep., 7, 1614, https://doi.org/10.1038/s41598-017-01673-9.

$\longrightarrow,-,-,-$, , and — 2018: The "bag breakup" spume droplet generation mechanism at high winds. Part I: Spray generation function. J. Phys. Oceanogr., 48, 2167-2188, https://doi.org/10.1175/JPO-D-17-0104.1.

Veron, F., 2015: Ocean spray. Annu. Rev. Fluid Mech., 47, 507-538, https://doi.org/10.1146/annurev-fluid-010814-014651.

— C. Hopkins, E. L. Harrison, and J. A. Mueller, 2012: Sea spray spume droplet production in high wind speeds. Geophys. Res. Lett., 39, L16602, https://doi.org/10.1029/2012GL052603.

Wright, C. W., and Coauthors, 2001: Hurricane directional wave spectrum spatial variation in the open ocean. J. Phys. Oceanogr., 31, 2472-2488, https://doi.org/10.1175/1520-0485(2001) 031<2472:HDWSSV $>2.0$.CO;2.

Wu, J., 1974: Evaporation due to spray. J. Geophys. Res., 79, 41074109, https://doi.org/10.1029/JC079i027p04107.

Zhang, J. A., R. F. Rogers, P. D. Reasor, E. W. Uhlhorn, and F. D. Marks, 2013: Asymmetric hurricane boundary layer structure from dropsonde composites in relation to the environmental vertical wind shear. Mon. Wea. Rev., 141, 3968-3984, https:// doi.org/10.1175/MWR-D-12-00335.1.

Zhao, D., Y. Toba, K. Sugioka, and S. Komori, 2006: New sea spray generation function for spume droplets. J. Geophys. Res., 111, C02007, https://doi.org/10.1029/2005JC002960. 